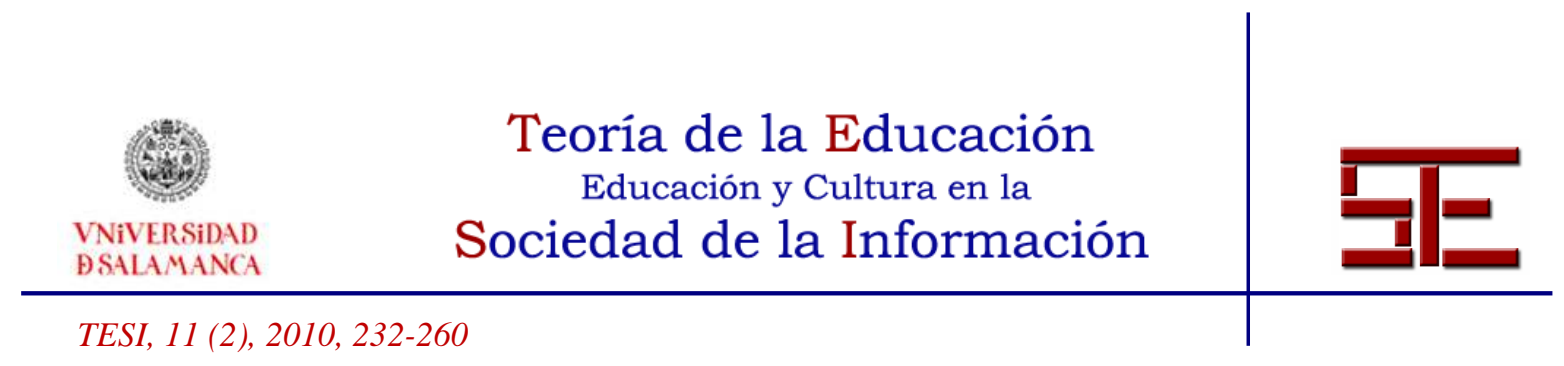

\title{
TELE-ENSEÑANZA A TRAVÉS DE INTERNET: LA PROTECCIÓN RA- DIOLÓGICA EN CIENCIAS DE LA SALUD
}

\section{Resumen}

La obtención de un proyecto interdepartamental subvencionado por el Ministerio de Educación y Ciencia español ha permitido crear materiales didácticos específicos de Protección Radiológica que se han publicado en varios manuales y cuadernos de actividades prácticas. Estas publicaciones han constituido la base del primer curso continuado sobre Protección Radiológica realizado a través de Internet en España (8 ediciones, durante los años 2000-2009). Durante el pregrado y el grado, la utilización de material didáctico digital apropiado determina el nivel de conocimientos que puede alcanzarse. La enseñanza multimedia y la tele-educación incrementan el interés de los alumnos en los temas más complicados y difíciles en Ciencias de la Salud. Posteriormente, cuando se abandona la Universidad y comienza la actividad profesional, la tele-enseñanza permite la formación continuada en las actualizaciones básicas sobre Protección Radiológica, permitiendo a todos los profesionales familiarizarse con medios y recursos que difícilmente podrán utilizar de forma personal y directa.

Palabras Clave: Radiología, tele-enseñanza, educación médica, protección radiológica, control de calidad. 


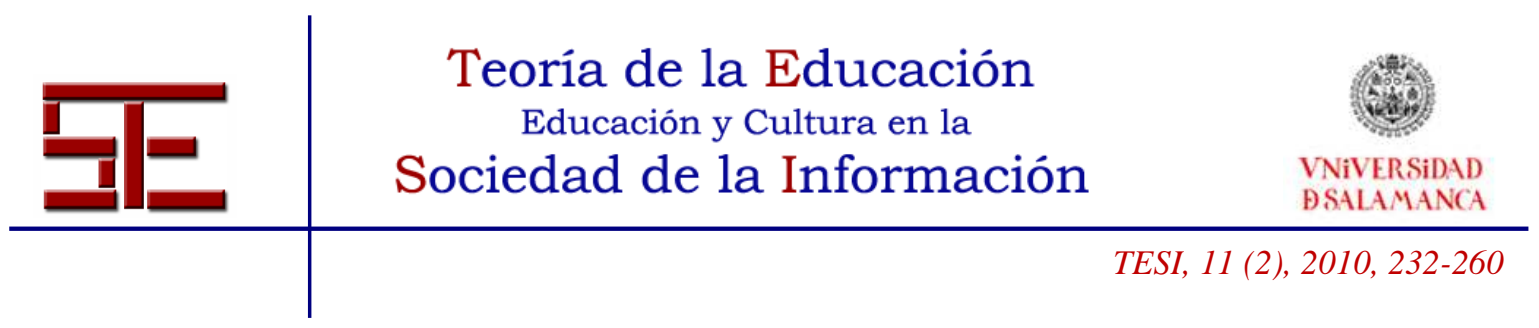

\title{
E-LEARNING THROUGH INTERNET: RADIOLOGICAL PROTECTION IN HEALTH SCIENCES
}

\begin{abstract}
:
The creation of an interdepartmental project subsidised by the Spanish Ministry of Education has made it possible to create teaching material for Radiological Protection, and led to the publication of several specific manuals and practical notebook. This material constitutes the working basis for the first continuous e-learning training course in Spanish via Internet on this subject (8 editions during the years 2000-2009). The use of appropriate teaching materials during the training cycles determines the level of knowledge that can be reached. Interactive multimedia teaching and e-learning increase interest in subjects that were previously regarded their as boring and difficult health science students'. When the students have finished their university studies, e-learning systems can provide continued professional training that achieves the basic goals of Radiological Protection, allowing professionals to acquaint themselves with this type of content, which they would usually fond difficult to do themselves.
\end{abstract}

Key Words: Radiology, e-learning, medical education, radiological protection, quality control. 


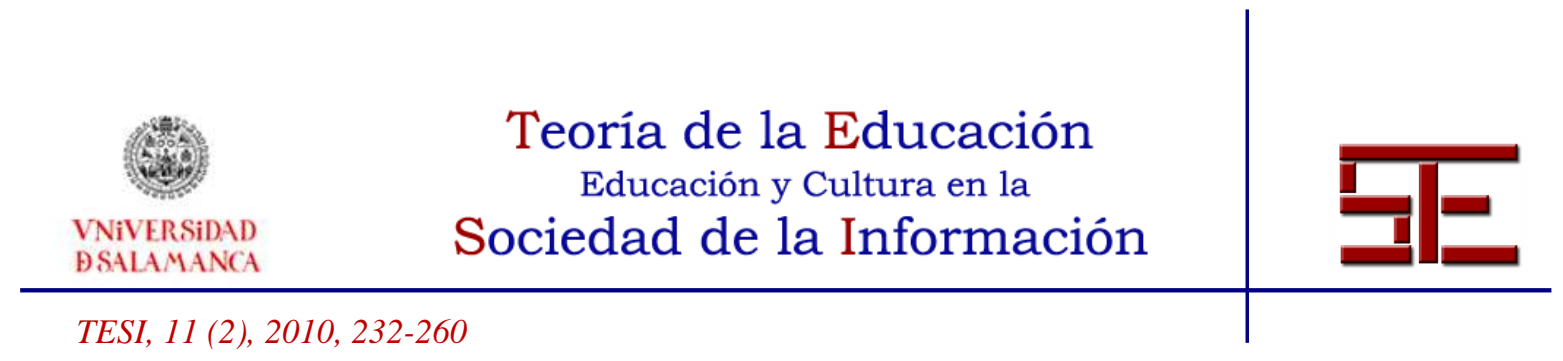

\title{
TELE-ENSEÑANZA A TRAVÉS DE INTERNET: LA PROTECCIÓN RA- DIOLÓGICA EN CIENCIAS DE LA SALUD
}

\author{
Miguel Alcaraz Baños \\ mab@um.es \\ Pablo Chico Sánchez \\ pchico@um.es \\ Yolanda Martínez Beneyto \\ yolandam@um.es \\ David Armero Barranco \\ darmero@um.es \\ Ana Belén Meseguer Henarejos \\ anabelen@um.es \\ Miguel Alcaraz Saura \\ miguel.alcaraz@um.es \\ Universidad de Murcia
}

\section{1.- INTRODUCCIÓN}

Los cambios que se están produciendo en el ámbito universitario español para su adaptación al Espacio Europeo de Educación Superior (EEES) traen como consecuencia una amplia serie de modificaciones en el modelo de enseñanza universitaria que se ha desarrollado hasta ahora. Se pretende alcanzar un rol distinto para la Universidad y para el docente. La labor del profesorado debe pasar de un papel más bien pasivo en el cual se limitaba a la impartición de clases magistrales a un rol más activo en el que se precisa de una mayor creatividad y utilización de nuevas herramientas que permitan al alumnado hacerse más participativo en el aula, fuera de ella, y en su propio proceso de aprendizaje. El profesorado debe reflexionar sobre su manera y forma de enseñanza, introduciendo nuevas estrategias formativas, y analizar su docencia con el fin de mejorarla.

En este sentido, y desde el año 1997 el Departamento de Radiología y Medicina Física de la Universidad de Murcia atraído por las ventajas de la enseñanza asistida por orde-

Miguel Alcaraz Baños, Pablo Chico Sánchez, Yolanda

$234 \quad \begin{gathered}\text { Martínez Beneyto, David Aremero Barranco, Ana Belén } \\ \text { Meseguer Henarejos y Miguel Alcaraz Saura }\end{gathered}$




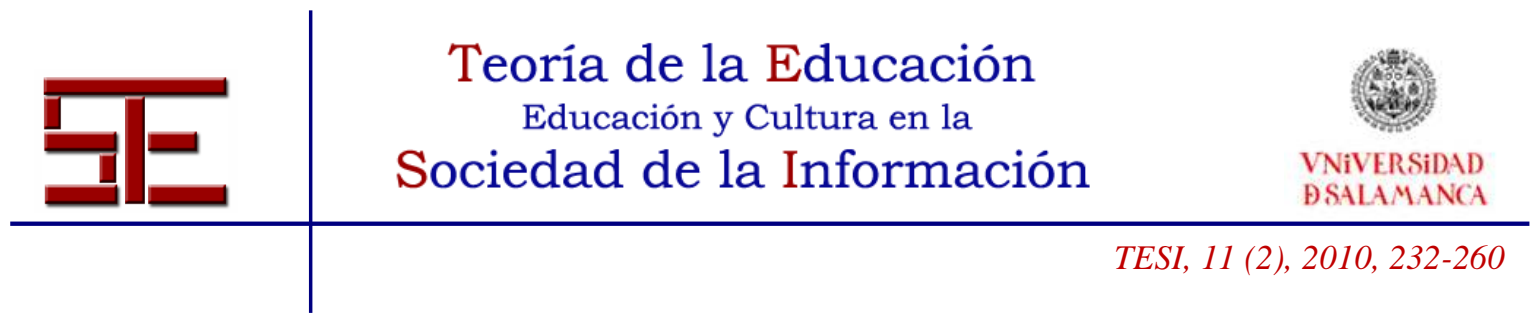

nador y la tele-enseñanza (Squire y Becker, 1975; Goldman y Blake, 1996), ha ido desarrollando cursos de formación interactiva, mediante metodologías activas, a diferentes niveles de formación académica y profesional, no solamente universitarios.

Como consecuencia de la Convocatoria de Proyectos de Cooperación entre Departamentos Universitarios y Departamentos de Enseñanza Secundaria, realizada por Resolución de la Secretaría General de Educación y Formación Profesional (BOE de 23 de Octubre de 1997), se obtuvo un proyecto de Cooperación que pretendía la "Elaboración de material didáctico de Protección Radiológica y Control de Calidad en Radiodiagnóstico para el alumnado de Técnico Superior en Imagen para el Diagnóstico”, ya que en aquel momento dicho material era prácticamente inexistente. El objetivo planteado era dotar al alumno de un material científicamente actualizado y didácticamente ameno. Para ello, debían presentarse adecuadamente los conceptos de criterios de calidad, dosis de radiación, radioprotección y sus implicaciones, facilitando el aprendizaje mediante el uso del ordenador.

Inmediatamente después, desde el año 2000, se han ido adaptando dichos materiales para la realización de diferentes ediciones de un curso de tele-enseñanza (e-learning), a través de Internet con los materiales y recursos originales que se han producido. Estos cursos se han ampliado a otros profesionales que también utilizan las radiaciones desde la perspectiva de la imagen diagnóstica médica, como son médicos, radiólogos, odontólogos, enfermeros, fisioterapeutas, técnicos superiores en higiene bucodental y técnicos superiores en imagen para el diagnóstico. El objetivo inicial planteado con la incorporación de estos sistemas "on-line" educativos es proporcionar al estudiante, en cada uno de los diferentes grados académicos al que pertenece, una puesta al día de los conocimientos en protección radiológica, de forma amena, entretenida y mediante métodos didácticos interactivos. En este trabajo se describe el análisis de los materiales originales producidos en Protección Radiológica, su evaluación inicial con los alumnos y el proceso de incorporación y desarrollo en los cursos de tele-enseñanza impartidos a través de Internet, para discutir las ventajas e inconvenientes que este sistema de enseñanza nos ha proporcionado durante las 8 ediciones anuales que hemos realizado.

\section{2.- MATERIAL Y MÉTODOS}

El protocolo de realización de este trabajo puede sintetizarse en tres fases diferentes: elaboración de los materiales didácticos especializados, evaluación docente de dicho material en dos cursos de estudiantes de Técnico Superior en Imagen para el Diagnóstico y desarrollo de los cursos de tele-enseñanza a través de Internet.

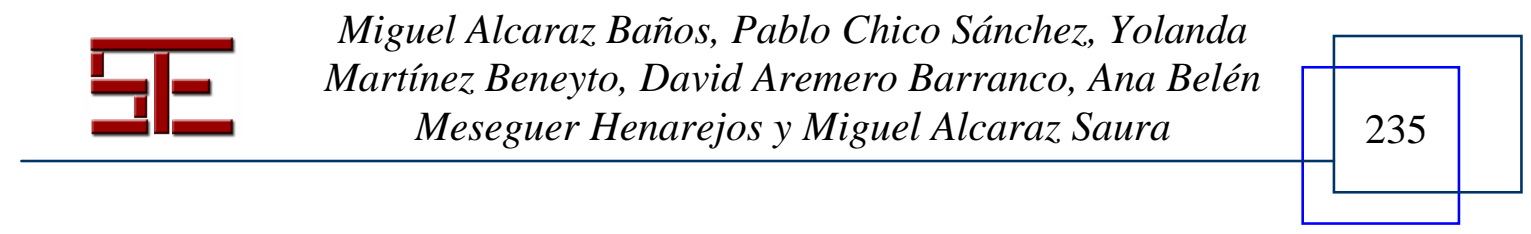




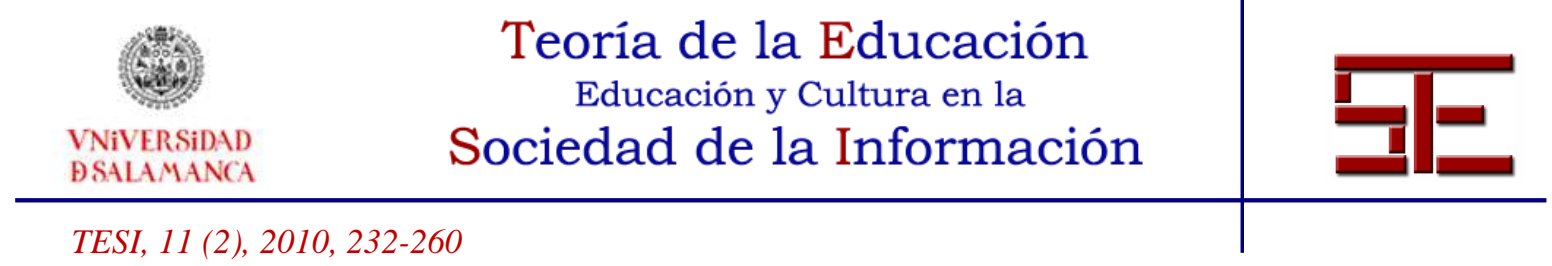

Fase I: Elaboración de materiales y recursos docentes.

Todo el profesorado del Área de Técnico Superior en Imagen para el Diagnóstico del Instituto de Formación Profesional "Juan de la Cierva" de la Comunidad Autónoma de la Región de Murcia y el de la Unidad Docente de Radiología y Medicina Física de la Universidad de Murcia, tras la obtención de una ayuda del Ministerio de Educación y Ciencia (BOE 23/10/1997), se han dedicado durante un período de 24 meses a la elaboración de materiales y recursos didácticos originales que no existían en lengua española. Para la elaboración de este material especializado y científicamente actualizado, se fueron realizando sesiones conjuntas de elaboración de los diferentes materiales originales, partiendo de los contenidos y recursos del Área de Radiología y Medicina Física, guiados por las premisas expuestas en los programas VALUE y ERPET de la Unión Europea. Se han utilizado los materiales elaborados durante diferentes cursos de formación específica realizados en la Universidad de Murcia y en

las publicaciones y materiales propios del Grupo de Investigación de Radiología Experimental de la Universidad de Murcia, tomando como base de partida el texto previamente publicado sobre Protección Radiológica en Radiodiagnóstico (Alcaraz y Genovés, 1996) (Figura 1).

A partir de estas primeras publicaciones, la actividad realizada por la Unidad Docente de Radiología y Medicina Física ha

MIGUEL ALCARAZ BAÑOS JOSÉ LUIS GENOVÉS GARCÍA

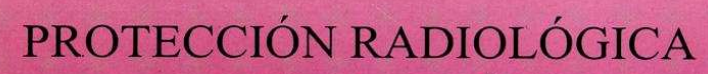
EN RADIODIAGNÓSTICO

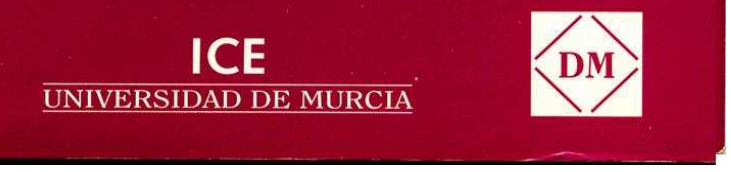

Fig.1: Material de partida en la elaboración de los nuevos recursos y materiales (Alcaraz y Genovés, 1995) seguido produciendo materiales que se han publicado a través del Servicio de Publicaciones de la Universidad de Murcia en colaboración con otras instituciones públicas (Ministerio de Educación y Ciencia, Consejo de Seguridad Nuclear y Comunidad Autónoma de la Región de Murcias), siguiendo las recomendaciones sobre formación en Protección Radiológica de la Unión Europea (European Commission, 2000).

Miguel Alcaraz Baños, Pablo Chico Sánchez, Yolanda Martínez Beneyto, David Aremero Barranco, Ana Belén Meseguer Henarejos y Miguel Alcaraz Saura 


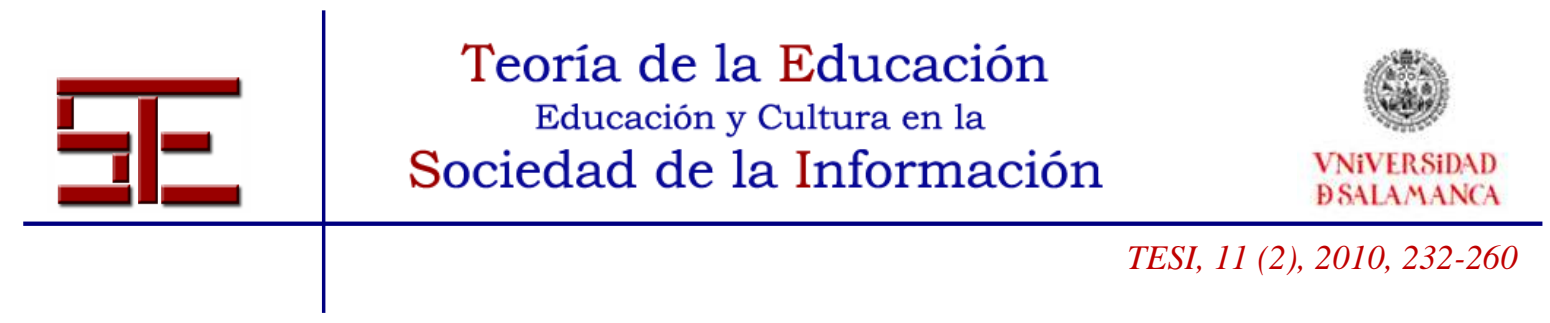

Fase II: Evaluación docente de los materiales y recursos docentes elaborados en estudiantes de Técnico Superior en Imagen para el Diagnóstico.

Los materiales y recursos propios elaborados se ofertaron y evaluaron en una muestra de alumnos que comprende un total de 60 estudiantes (30 alumnos por año, de cada una de las dos promociones consecutivas estudiadas) de $2^{\circ}$ curso de Técnico Superior en Imagen para el Diagnóstico, procedentes del Instituto de Formación Profesional "Juan de la Cierva" de la Comunidad Autónoma de la Región de Murcia. La selección de la muestra se realizó mediante muestreo intencional, estando representado el $100 \%$ de la población de procedencia. En todos los casos se realizaron tests de evaluación del aprendizaje del alumnado, auto-evaluación del alumnado y del profesorado y una coevaluación conjunta profesorado-grupo de alumnos. Durante el primer año se realizaron los test para determinar cuantitativamente los parámetros básicos y los niveles de conocimientos de partida. Durante el siguiente curso académico, se utilizaron los medios ya confeccionados y se procedió al análisis para la determinación de las posibles modificaciones de las variables estudiadas.

\section{Fase III: Cursos de Tele-enseñanza a través de Internet.}

Desde el año 2000 se han llevado a cabo 8 ediciones anuales del curso de Promoción Educativa sobre "Protección Radiológica y Garantía de Calidad en Radiodiagnóstico. Curso de tele-enseñanza", con carácter de formación continuada organizado por el Grupo de Investigación de Radiología Experimental de la Universidad de Murcia. El curso tiene carácter "on-line", realizado exclusivamente a través de Internet, donde el alumno es asesorado mediante tutorías sincrónicas y asincrónicas que permiten el seguimiento del alumno durante el curso, así como un proceso de auto-evaluación del alumno y de evaluación del alumno por el profesor.

Utilizando Internet como medio de trabajo y siguiendo los estándares del formato HTML, se desarrolló un portal de acceso web que ubicamos en un servidor independiente dentro del Grupo de Investigación en Radiología Experimental. El servidor que se empleó fue un simple ordenador de la serie Veriton (Acer Computer Ibérica, España), equipado con un procesador Pentium IV (Intel Corporation, California), $256 \mathrm{MB}$ de RAM, y 40 GB de disco duro. Dotado de sistema operativo Windows XP (Microsoft Corporation, Redmond, Washington) y un monitor en color 800x600 (Acer Computer Ibérica, España). El equipo se encuentra conectado a la Red LAN de la Universidad de Murcia, dependiente de REDIRIS, la Red Española de I+D que provee de acceso a Internet a los centros oficiales, a través de la cual el servidor tiene salida a Internet. ATICA (Servicio de Informática de la Universidad de Murcia), nos proporcionó el DNS ó nombre de dominio http://radiologia.um.es que redirige todas las peticiones de red de esa dirección directamente a nuestro servidor. De ésta forma el acceso es fácil desde

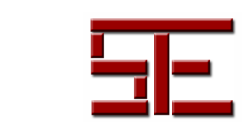

Miguel Alcaraz Baños, Pablo Chico Sánchez, Yolanda Martínez Beneyto, David Aremero Barranco, Ana Belén Meseguer Henarejos y Miguel Alcaraz Saura 


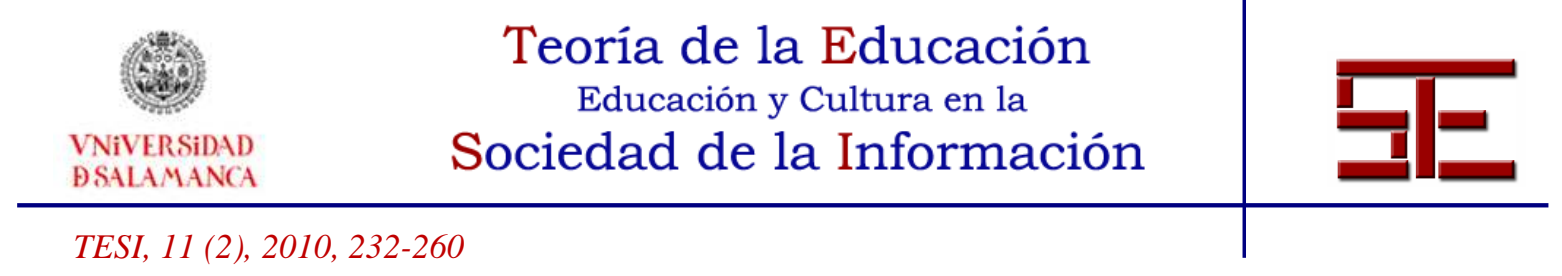

cualquier ordenador conectado a Internet y tiene la suficiente independencia para trabajar de forma autónoma.

Como Software que sirviera las páginas web, utilizamos la distribución Apache (Apache Software Foundation) en su versión 2.0, ubicando dentro del servidor los contenidos en formato HTML que habíamos diseñado. Los contenidos del portal de acceso fueron desarrollados de acuerdo a los estándares para el lenguaje HTML propuestos por la W3C (World Wide Web Consortium) en su versión HTML 4.01.

Todo esto nos permitió impartir el primer curso de tele-enseñanza en Protección Radiológica y Garantía de Calidad en Radiodiagnóstico que se ha desarrollado a nivel nacional a través de Internet y que se desarrolló gracias a una subvención específica del Consejo de Seguridad Nuclear en su convocatoria I+D+i de 2001 (MMC/SUBV/UMU/064/2003). El curso ofreció dos posibilidades en su primera edición: presencia física para aquellos alumnos matriculados que procedía en la Facultad de Medicina de la Universidad de Murcia y Desarrollo Virtual exclusivo para todos aquellos que no lo eran. A partir de la segunda edición, el curso fue exclusivamente virtual, a través de Internet.

El curso tiene la acreditación de Curso de Promoción Educativa de la Universidad de Murcia y ha obtenido la acreditación de Interés científico-sanitaria Regional otorgada por la Consejería de Sanidad de la Comunidad Autónoma de la Región de Murcia. Para los alumnos de Medicina tiene carácter obligatorio por acuerdo de Junta de Facultad, mientras que para el resto de alumnos de Ciencias de la Salud están acreditados como Créditos de Libre Configuración por aprobación de sus Juntas de Centro. En estos momentos el curso y sus materiales están expuestos en la dirección de la Universidad de Murcia (http://webs.um.es/mab/miwiki/doku.php?id=docencia) con acceso libre y gratuito, aunque sus ediciones oficialmente sólo se realizan de octubre a enero, con una duración de 3,5 meses y con una valoración de 115 horas lectivas, actualmente de 6 ECTS.

Tras la realización de cada edición del curso se realiza una encuesta docente a alumnos y profesores para valorar el desarrollo del mismo y determinar las posibles modificaciones e incorporaciones para la siguiente edición.

\section{3.- RESULTADOS}

\section{Fase I: Elaboración del material docente}

Nuestros resultados pusieron de manifiesto una muy escasa bibliografía en español, carente de iconografía básica, y ausencia de material didáctico teórico y de materiales y

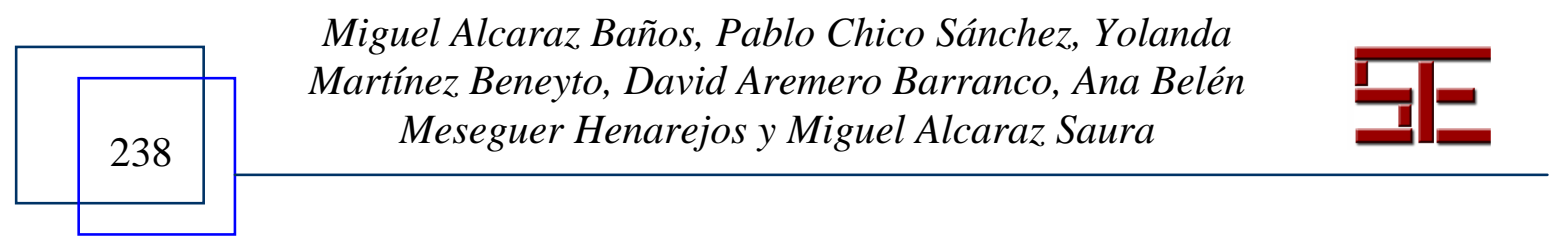




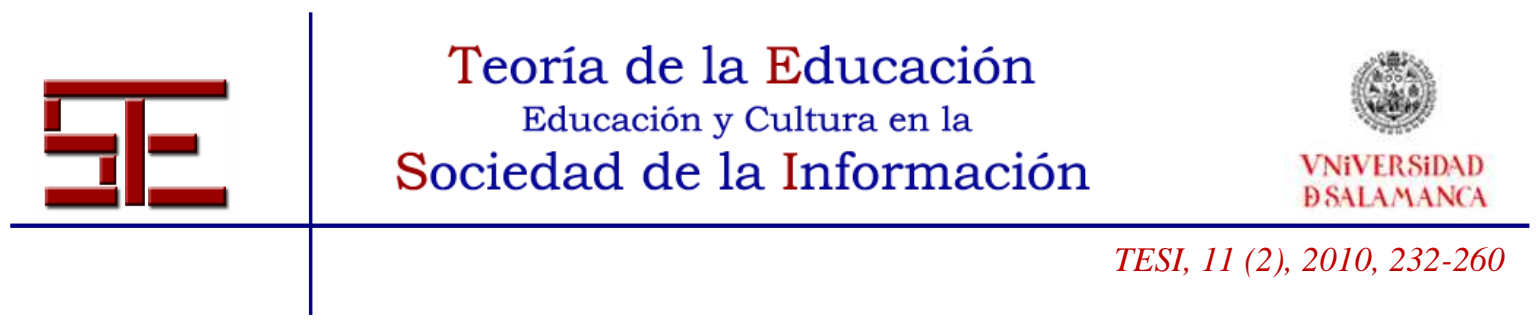

recursos para las actividades prácticas en Protección Radiológica incluidas en los diferentes ciclos formativos durante la primera evaluación realizada en 1997-98.

Tras la obtención de la Ayuda del Ministerio de Educación y Ciencia (BOE de 23/10/1997), se elaboraron materiales y recursos didácticos mediante un proyecto de Cooperación entre el Área de Radiología y Medicina Física de la Universidad de Murcia y la Familia Profesional del Instituto de Enseñanza Secundaria "Ingeniero de la Cierva" de Murcia, que permitieron la publicación de los primeros materiales en un libro titulado "Bases Físicas y Biológicas del Radiodiagnóstico Médico. Cuaderno de Actividades Prácticas (Alcaraz, 2003a) (Figura 2), del que se han realizado 2 ediciones diferentes para mantener su actualización y cuatro reimpresiones hasta estos momentos. El objetivo fundamental era dotar al alumno de un material científicamente actualizado y didácticamente ameno que pudiera ser utilizado en el aula. Para ello, se han debido presentar adecuadamente los conceptos de criterios de calidad, dosis de radiación, radioprotección y sus

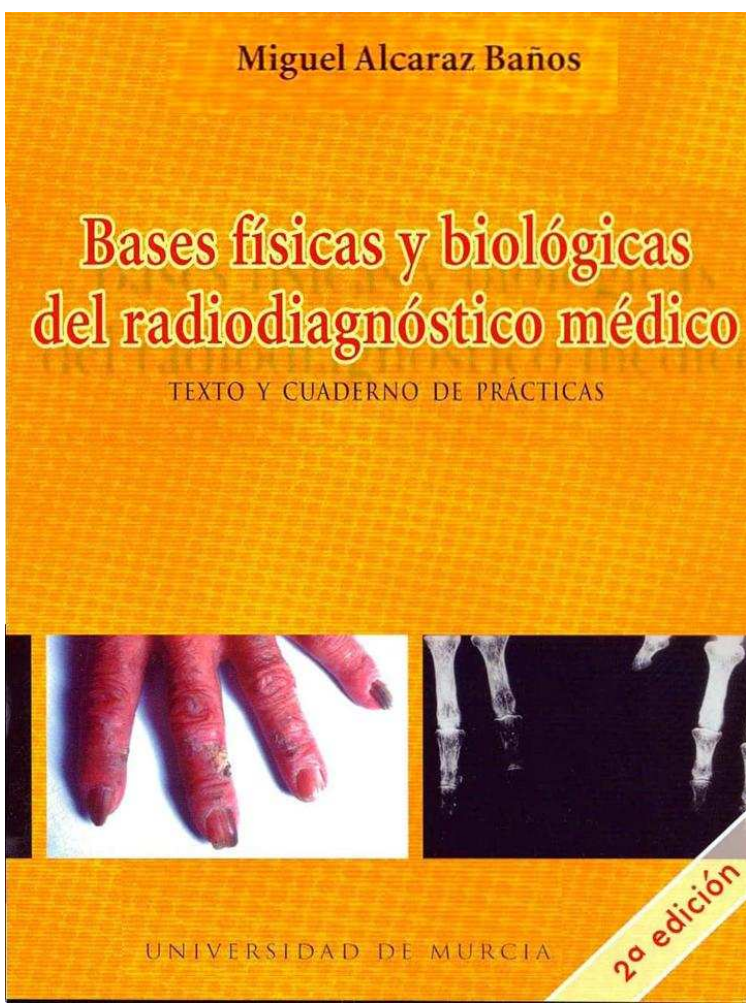

Fig.2: Materiales generados consecuencia de la subvención del Ministerio de Educación y Ciencia (Alcaraz 2003). implicaciones; facilitando el aprendizaje mediante el uso del ordenador. En el manual, se han incluido actividades prácticas con los tests de control de calidad más importantes en un programa de Garantía de Calidad en Radiodiagnóstico y preguntas de autoevaluación mediante test objetivo de opciones múltiples al final del texto, en donde no se incluyen las respuestas correctas siguiendo los consejos de los expertos en pedagogía de la Universidad de Murcia (UMU); todo el material ha sido publicado en versión digital en diferentes formatos. Los contenidos básicos teóricos y prácticos del trabajo se corresponden con los cursos denominados de capacitación para Operadores de Instalaciones radiactivas con fines de diagnóstico médico/dental necesarios para manipular equipos de radiodiagnóstico médico o dental y se han realizado siguiendo las recomendaciones de formación en Protección Radiológica de la Unión Europea (European Commission, 2000).

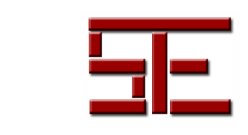

Miguel Alcaraz Baños, Pablo Chico Sánchez, Yolanda Martínez Beneyto, David Aremero Barranco, Ana Belén Meseguer Henarejos y Miguel Alcaraz Saura 


\begin{tabular}{ccc}
$c$ & $\begin{array}{c}\text { Teoría de la Educación } \\
\text { Educación y Cultura en la } \\
\begin{array}{l}\text { VNiVERSIDAD } \\
\text { DSALAMANCA }\end{array}\end{array}$ \\
\hline TESI, $11(2), 2010,232-260$ & Sociedad de la Información & \\
\hline
\end{tabular}

La actividad docente desarrollada ha generado con posterioridad nuevos materiales y recursos que son imprescindibles para la realización de los cursos de formación "online" y que, con este objetivo específico, se han desarrollado. Así, se han obtenido dos ayudas de proyectos públicos competitivos en las convocatorias de I+D del Consejo de Seguridad Nuclear que han permitido la realización y publicación de dos textos editados conjuntamente con la Universidad de Murcia que se han incorporado a los cursos impartidos a través de Internet: "La evolución de la Protección Radiológica dental en España (Alcaraz, 2005) (CSN, MMC/SUBV/UMU/064/2003) y "La Radiología Dental en España" (Alcaraz, 2008) (CSN, BOE de 24/09/2007, Resol BOE nº 16 de 05/03/2007) (Figura 3).
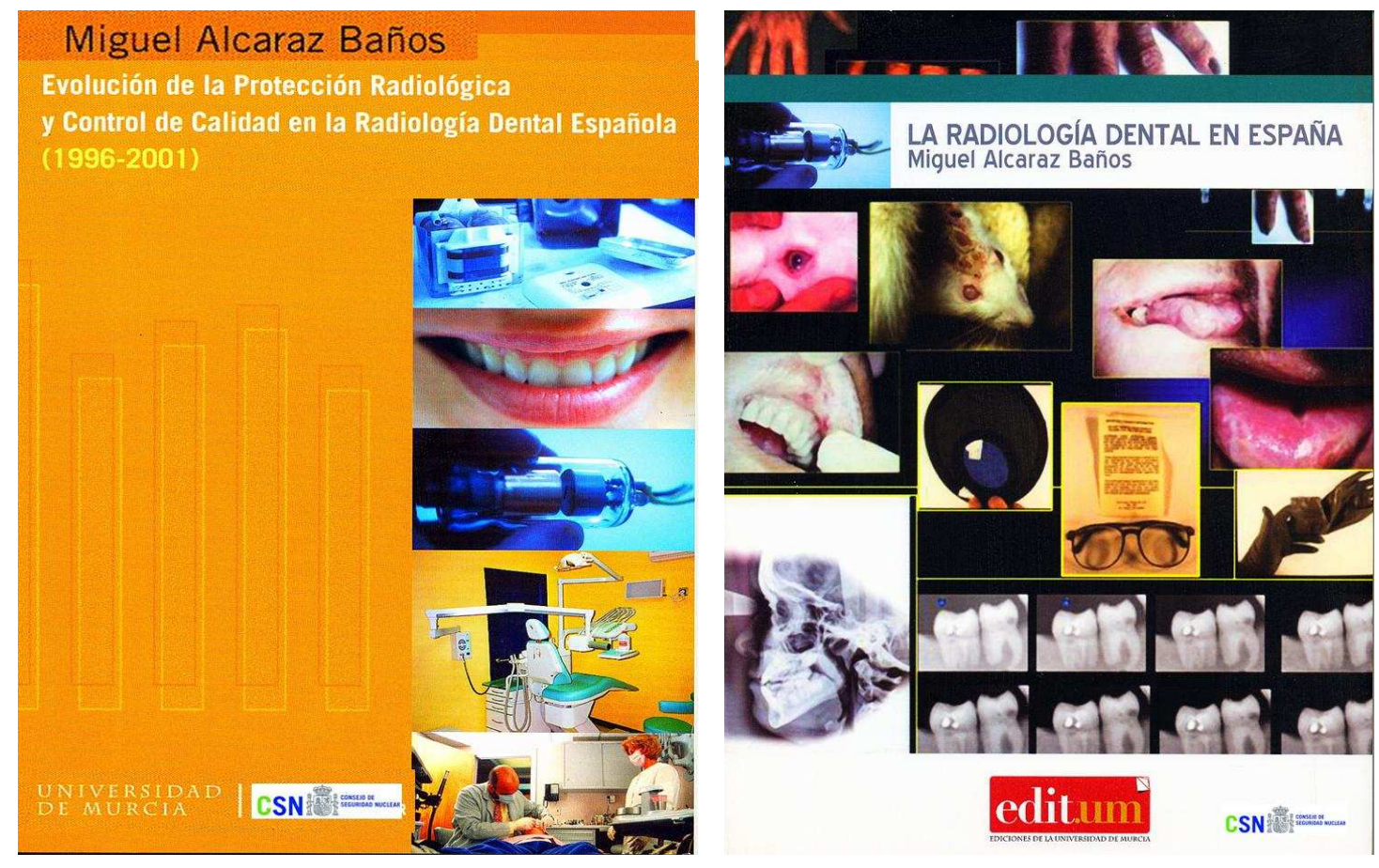

Fig.3: Libros editados mediante la subvención del Consejo de Seguridad Nuclear y que forman parte de los contenidos e iconografía desarrollada para el curso de tele-enseñanza (Alcaraz, 2005, 2008).

Como consecuencia de esta labor docente, hemos incorporado la Protección Radiológica como una de las líneas de investigación de nuestro Grupo de Radiología Experimental, generando diferentes tipos de publicaciones que se han ido integrando dentro de los recursos docentes en los cursos de tele-enseñanza a través de Internet tanto desde el punto de vista de la Protección Radiológica y Control de Calidad en Radiodiagnóstico

Miguel Alcaraz Baños, Pablo Chico Sánchez, Yolanda Martínez Beneyto, David Aremero Barranco, Ana Belén Meseguer Henarejos y Miguel Alcaraz Saura

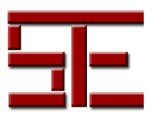




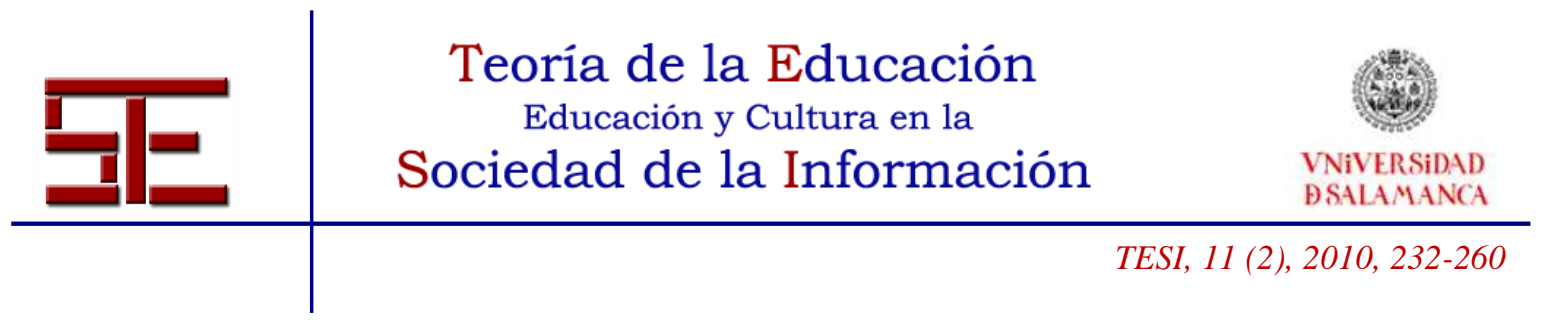

(Alcaraz et al., 2000, 2004a, 2004b, 2006a, 2009a, 2009b, 2009c, 2009d, 2010; Martínez-Beneyto et al., 2004, 2007, 2008), como desde la Protección Radiológica frente al daño biológico inducido por la radiación ionizante (Alcaraz et al., 2009d, Castillo et al., 2002; Navarro et al., 2004, Del Baño et al., 2006; Sánchez-Campillo et al., 2009) (Figuras 4 y 5 ).

Por último, se obtuvo una ayuda de la Comunidad Autónoma de la Región de Murcia en colaboración con el Fondo Social Europeo (Registro de la CARM 2S118 N 200500067774) para la "Elaboración de diversos materiales didácticos destinados a Cursos de Supervisores de Instalaciones Radiactivas para el Centro Nacional de Formación Ocupacional de Cartagena", que está en fase de publicación, y algunos de cuyos contenidos se han incorporado también a los cursos realizados a través de Internet.

Durante estos años, tras una de las actualizaciones de los planes de estudio en Ciencias de la Salud, se han ido incorporado algunos de estos contenidos a otras enseñanzas de la Universidad de Murcia, constituyendo los textos base en la asignatura de Protección Radiológica en Odontología (impartida en $4^{\circ}$ Curso de la Licenciatura de Odontología), y en la asignatura de Biofísica Radiológica (impartida en $2^{\circ}$ curso de Enfermería), y un material de apoyo para los estudiantes de Medicina en los apartados respectivos de la asignatura de "Radiología y Medicina Física" ( $3^{\circ}$ curso de la Licenciatura en Medicina). Desde el año 2000, estos materiales son los que se han empleado en los cursos de Protección Radiológica realizados a través de Internet.

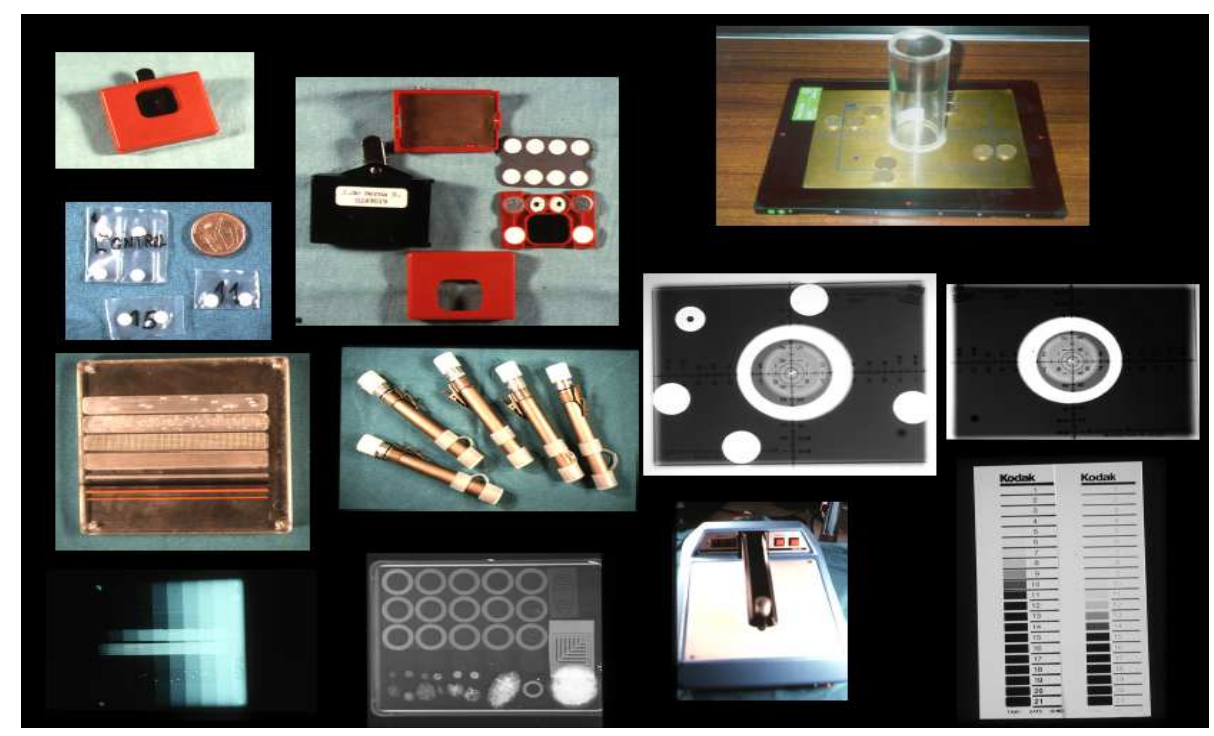

Fig.4: Iconografía específica diseñada sobre test y ensayos de control de calidad y detectores de radiación ionizante elaborados específicamente para el desarrollo del curso de teleenseñanza.

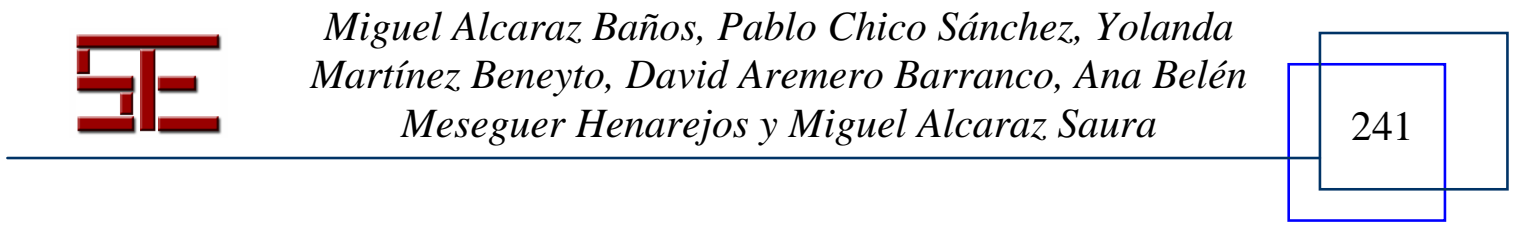




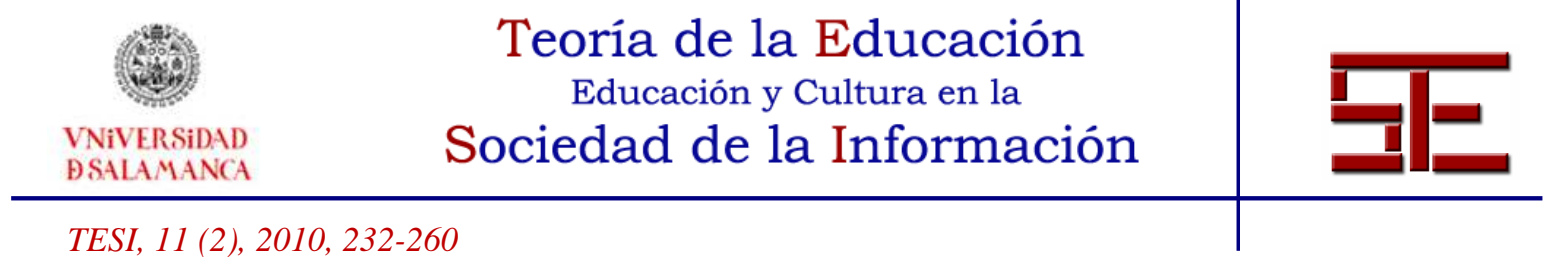

\section{Fase II: Formación a estudiantes de Técnico Superior en Imagen por Diagnóstico}

Tras la elaboración de estos materiales didácticos, se llevó a cabo su ensayo y análisis en el $2^{\circ}$ curso de Técnico Superior en Imagen para el Diagnóstico con la finalidad de determinar la utilidad de dicho material.

El análisis de los resultados obtenidos muestra un aumento en el grado de comprensión de los temas y del interés por los mismos, en opinión de los alumnos, con un incremento de demandas de orientación hacia el profesor y la solicitud de mayor cantidad de materiales didácticos del mismo tipo de los ya confeccionados. Igualmente, se obtuvieron valoraciones positivas de los profesores, describiéndose mayor motivación y adquisición de conocimientos al aproximar al docente al medio asistencial y profesional. Sobresale el aspecto de tener un material científico actualizado en castellano y dirigido básicamente a la actividad profesional en donde deberá ingresar el estudiante al finalizar sus estudios. Los resultados de la coevaluación muestran también un incremento en el interés del alumno por los temas tratados, así como en el cumplimiento de las tareas encomendadas junto con una mejor actitud del grupo frente al profesor. El análisis de las calificaciones finales en los dos grupos estudiados no mostró diferencias estadísticamente significativas (Figuras 6). Parcialmente, algunos de estos resultados se han presentado a diversos congresos y reuniones científicas (Alcaraz et al., 2002a, 2002b).

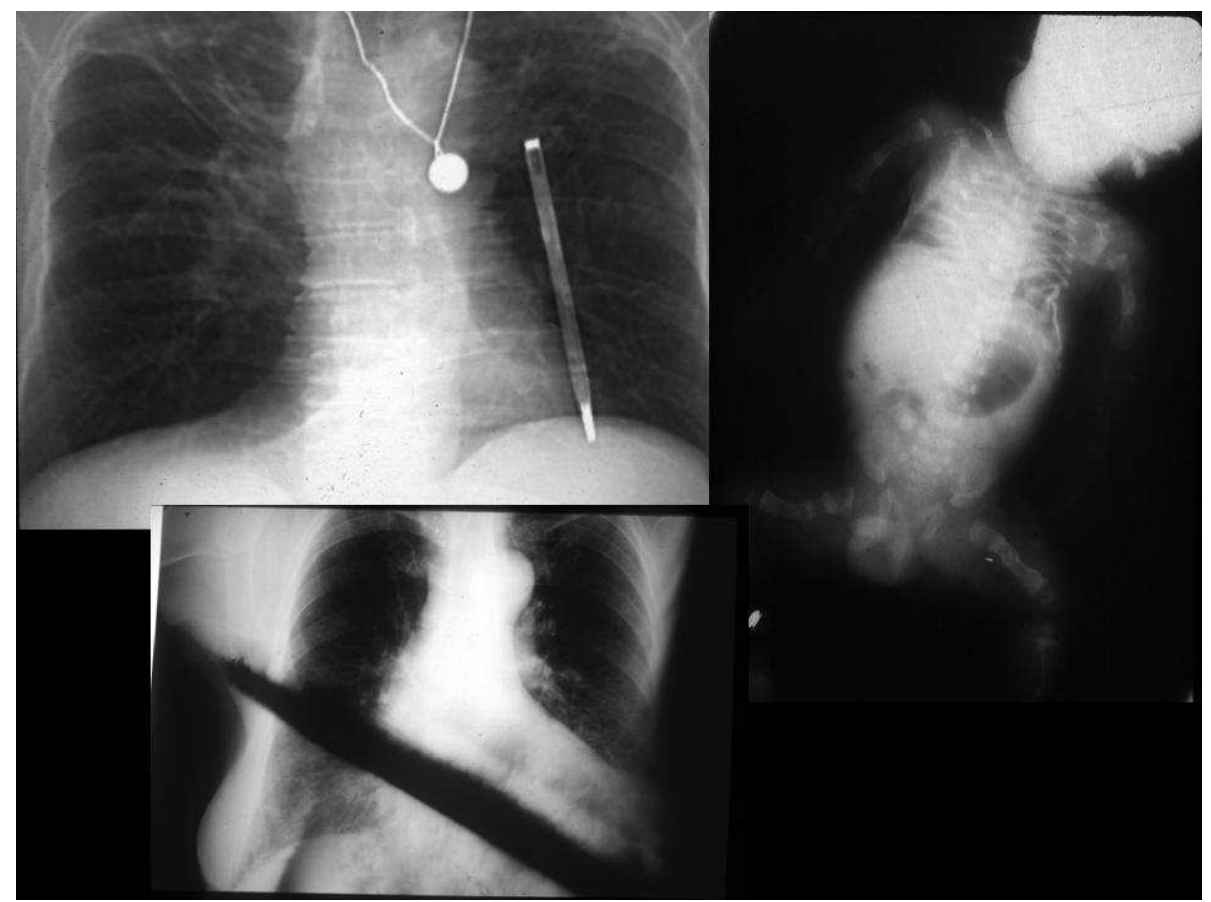

Fig.5: Iconografía sobre errores y defectos habituales desarrolladas para los cursos de tele-enseñanza: elementos metálicos, joyas, exposiciones corporales totales ("niñografía”) y entrada de luz en los chasis. radiográficos....

Miguel Alcaraz Baños, Pablo Chico Sánchez, Yolanda

$242 \quad \begin{gathered}\text { Martínez Beneyto, David Aremero Barranco, Ana Belén } \\ \text { Meseguer Henarejos y Miguel Alcaraz Saura }\end{gathered}$


Tabla III. AUTOEVALUACIÓN FORMATIVA DE LOS ALUMNOS.

\begin{tabular}{|c|c|c|}
\hline ASPECTOS A CONSIDERAR & & $\begin{array}{l}\text { BALANCE GENERAL U } \\
\text { OBSERVACIONES }\end{array}$ \\
\hline Aporto inclativas al grupo & $\mathbf{0}$ & \\
\hline Escojo la tarea que debla reelizar & O & \\
\hline Me ha reaultado fach la tarea & $\mathbf{O}$ & \\
\hline He aprendido of manejo de la técnica de obtención de datos & B & \\
\hline He ayudado a obtener conclusiones preclesas en el grupo & B & \\
\hline He mejorado la coimprensión eobre of tema & $M$ & \\
\hline Me he integrado en el grupo & $\mathbf{B}$ & \\
\hline Me ha reaultedo interesente ef trabajo & $\mathbf{M}$ & \\
\hline He hablado con ol profesor & $\mathbf{s}$ & \\
\hline He trabajado regularmente y con ciarto método y esfuerzo & B & \\
\hline 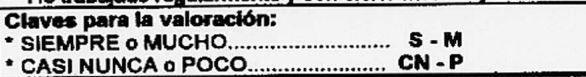 & * OC & NTE O BASTANTE: O-B \\
\hline
\end{tabular}

Tabla IV. EVALUACIÓN FORMATIVA - GRUPOS DE ALUMNOS

(COEVALUACIÓN)

\begin{tabular}{|c|c|}
\hline ASPECTOS A CONSIDERAR & $\begin{array}{l}\text { BALANCE GENERAL U OBSERVACIONES } \\
\text { DESTACABLES }\end{array}$ \\
\hline OBJETIVOS: Grado de intert́s y esfuerzo & $\begin{array}{l}\text { Bueno. En general es un grupo que responde cuando se le } \\
\text { pide un esfuerzo }\end{array}$ \\
\hline $\begin{array}{l}\text { CONTRIBUCION INDIVIDUAL DE LOS } \\
\text { MIEMBROS AL GRUPO }\end{array}$ & $\begin{array}{l}\text { Existen alumnos/as muy participativos mientras que en otros } \\
\text { es necesaria una dedicacłón especial. }\end{array}$ \\
\hline PARTICIPACION COMUNICATIVA & Igual que al partado anterior \\
\hline $\begin{array}{c}\text { CLIMA: carencla de actividad, apatla, amblente } \\
\text { alentador }\end{array}$ & Amblente alentador que favorece la dedicación y enseftanza. \\
\hline TOMA DE DECISIONES & $\begin{array}{l}\text { El amblente anterior favorece el trabajo conjunto, y el diseno } \\
\text { de actvidades. }\end{array}$ \\
\hline $\begin{array}{l}\text { EVALUACION: desánirno, interés por mejorar, } \\
\text { eficacla }\end{array}$ & Siempre se ha detectado un interés por mejorar y aprender. \\
\hline $\begin{array}{l}\text { RELACION CON EL PROFESOR: } \\
\text { indiferencla, demanda de orientación... }\end{array}$ & Positiva, con demanda de orientación. \\
\hline EXCLUSIONES DEL GRUPO & No existen, sólo algunos/as menos participativos. \\
\hline ASPECTOS & \\
\hline
\end{tabular}

Tabla V. AUTOEVALUACIÓN DE LA TAREA DEL PROFESOR

\begin{tabular}{|c|c|}
\hline ASPECTOS A CONSIDERAR & $\begin{array}{l}\text { BALANCE GENERAL U OBSERVACIONES } \\
\text { DESTACABLES. Final de evaluación (y casi final } \\
\text { del curso) }\end{array}$ \\
\hline $\begin{array}{l}\text { GRADO DE DIFICULTAD DEL PROCESO DE } \\
\text { TRABAJO }\end{array}$ & $\begin{array}{l}\text { En ocastones ha sido complicado que el alumnado } \\
\text { comprendlera por la escasez de material lconográfico. }\end{array}$ \\
\hline $\begin{array}{l}\text { GRADO DE ADECUACIONDE LOS } \\
\text { PROCEDIMIENTOS ESCOGIDOS. }\end{array}$ & Bueno \\
\hline RELACIÓN CON LOS ALUMNOS. & Muy posittiva y fructifera. \\
\hline RECURSOS DIDÁCTICOS & $\begin{array}{l}\text { Se ha utilizado todo el material de dotación del ciclo } \\
\text { ralativo a protección radiologica y garantia de calidad, } \\
\text { asi como transparencias de elaboración propia, y videos } \\
\text { del Consejo de Seguridad Nuclear. } \\
\end{array}$ \\
\hline RELACIÓN EXTRAESCOLAR & Positiva \\
\hline CUMPLIMIENTO DE LOS OBJETIVOS. & $\begin{array}{l}\text { Se consideran cumplidos, pero mejorables con mís } \\
\text { abundancia de material diddactico. }\end{array}$ \\
\hline SASPECTOS & \\
\hline
\end{tabular}

Fig.6: Aspectos más significativos de los resultados de la autoevaluación de los alumnos, de la coevaluación alumnos-profesores y autoevaluación de la tarea realizada por el profesor.

\section{Fase III: Cursos de Tele-enseñanza a través de Internet.}

Desde el año 2000 se están realizando sucesivas ediciones del Curso de "Protección Radiológica y Garantía de Calidad en Radiodiagnóstico. Curso de Tele-enseñanza", como curso de Promoción Educativa organizado por la Universidad de Murcia, en don-

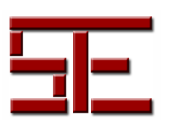

Miguel Alcaraz Baños, Pablo Chico Sánchez, Yolanda Martínez Beneyto, David Aremero Barranco, Ana Belén Meseguer Henarejos y Miguel Alcaraz Saura 


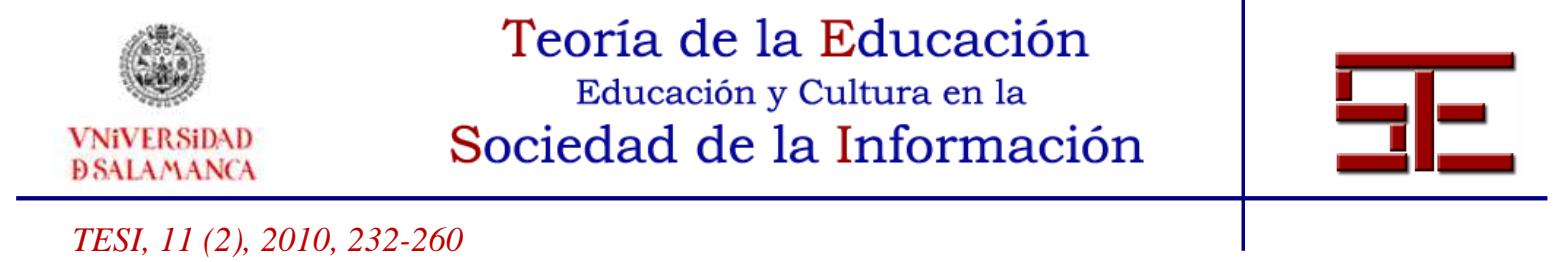

de se realiza la difusión de los contenidos en dos diferentes ámbitos de interés: a) en los profesionales que utilizan las radiaciones ionizantes para la obtención de imágenes diagnósticas (médicos, radiólogos, radiofísicos, odontólogos, técnicos especialistas en radiodiagnóstico, diplomados universitarios en enfermería, diplomados universitarios en fisioterapia y técnicos superiores en higiene buco-dental); y b) en profesionales sanitarios que tienen la obligación legal de conocer el riesgo biológico de la radiaciones ionizantes, aún cuando no sean directamente quienes obtienen las imágenes diagnósticas (médicos, auxiliares de enfermería, administrativos y celadores de los servicios de radiología, estudiantes de Ciencias de la Salud (medicina, odontología, enfermería y fisioterapia).

Para garantizar la privacidad de los contenidos se asignó a cada uno de los alumnos una pareja de usuario-clave de acceso que nos permitiera por una parte controlar que únicamente los alumnos matriculados en el curso pudieran descargar los contenidos; y por otra, realizar un control exhaustivo de las entradas que realiza para controlar el correcto aprovechamiento de los contenidos ofertados (Figura 7). La página principal esta dividida en las siguientes áreas: Características del curso; Índice de contenidos; Descarga de contenidos; Publicaciones; Enlaces interesantes; Ejercicios de autoevaluación; Contenidos Multimedia así como la presencia de un Tablón de Anuncios continuamente actualizado junto con un enlace explicativo para la primera vez que se accede al curso (Figuras 8 y 9). En estos momentos, el curso se encuentra accesible, abierto y gratuito en una página dentro de las páginas de la Universidad de Murcia en la dirección http://webs.um.es/mab/miwiki/doku.php?id=docencia, en donde pueden observarse todas las características actuales del mismo (Figura 10), aunque sus ediciones oficiales sólo se realizan tras la aprobación oficial del Servicio de Promoción Educativa de la Universidad de Murcia.

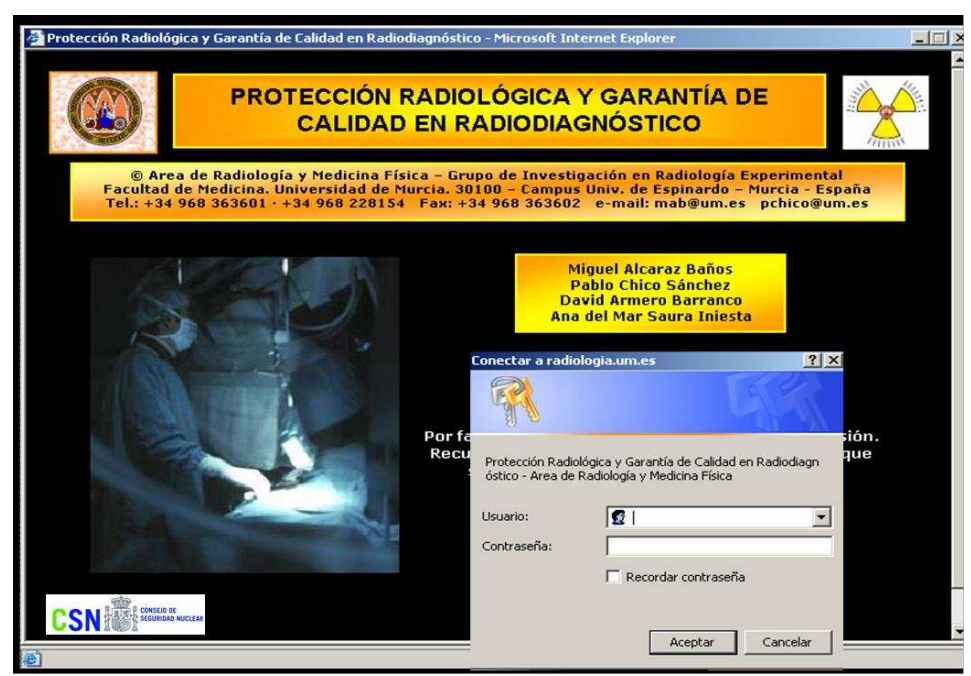

Fig.7: Página de entrada al curso con solicitud de claves de acceso al mismo.

Miguel Alcaraz Baños, Pablo Chico Sánchez, Yolanda

$244 \quad \begin{gathered}\text { Martínez Beneyto, David Aremero Barranco, Ana Belén } \\ \text { Meseguer Henarejos y Miguel Alcaraz Saura }\end{gathered}$




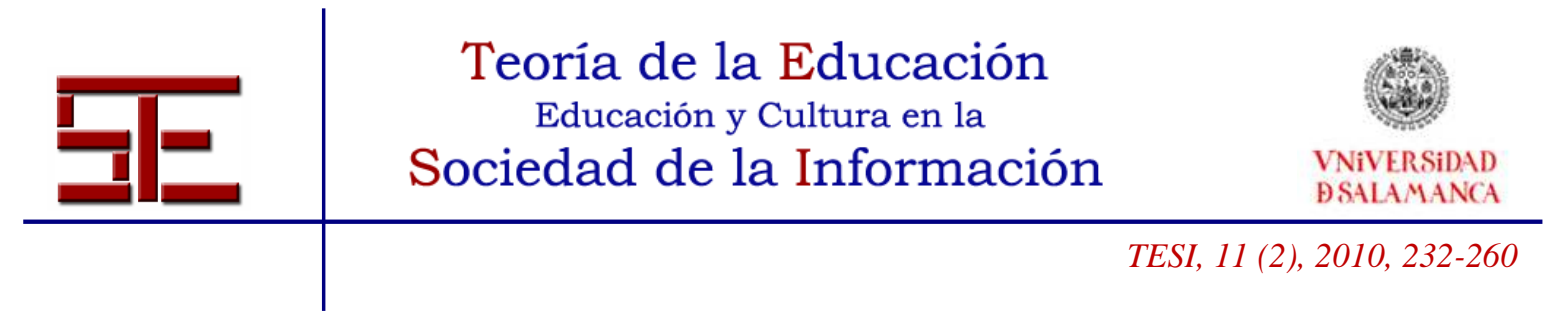

Este curso de tele-enseñanza ha sido el primer curso de Protección Radiológica y Control de Calidad impartido en España mediante este método interactivo "on-line" (Alcaraz et al, 2002a). Sin embargo, cabría destacar que el primer año de realización del mismo, y debido a la inseguridad y quizás falta de información por parte del alumnado de éste sistema de enseñanza, se decidió llevar a cabo el curso tanto de forma presencial, en la Facultad de Medicina, como virtual a través de Internet.

Hasta estos momentos, 2.123 alumnos han realizado este curso en sus diferentes ediciones con matrícula oficial de la Universidad de Murcia. Desde su primera edición (2000) han ido sucediéndose diferentes modificaciones en el desarrollo del mismo, fundamentalmente inducidas por el desarrollo de las conexiones a Internet (telefonía, modem, ADSL, LAN), las posibilidades de utilización del ordenador y de Internet por parte de los alumnos y las posibilidades de incrementar las descargas de vídeos y programas ejecutables, chats o foros a través de los servidores ftp de la Universidad de Murcia.

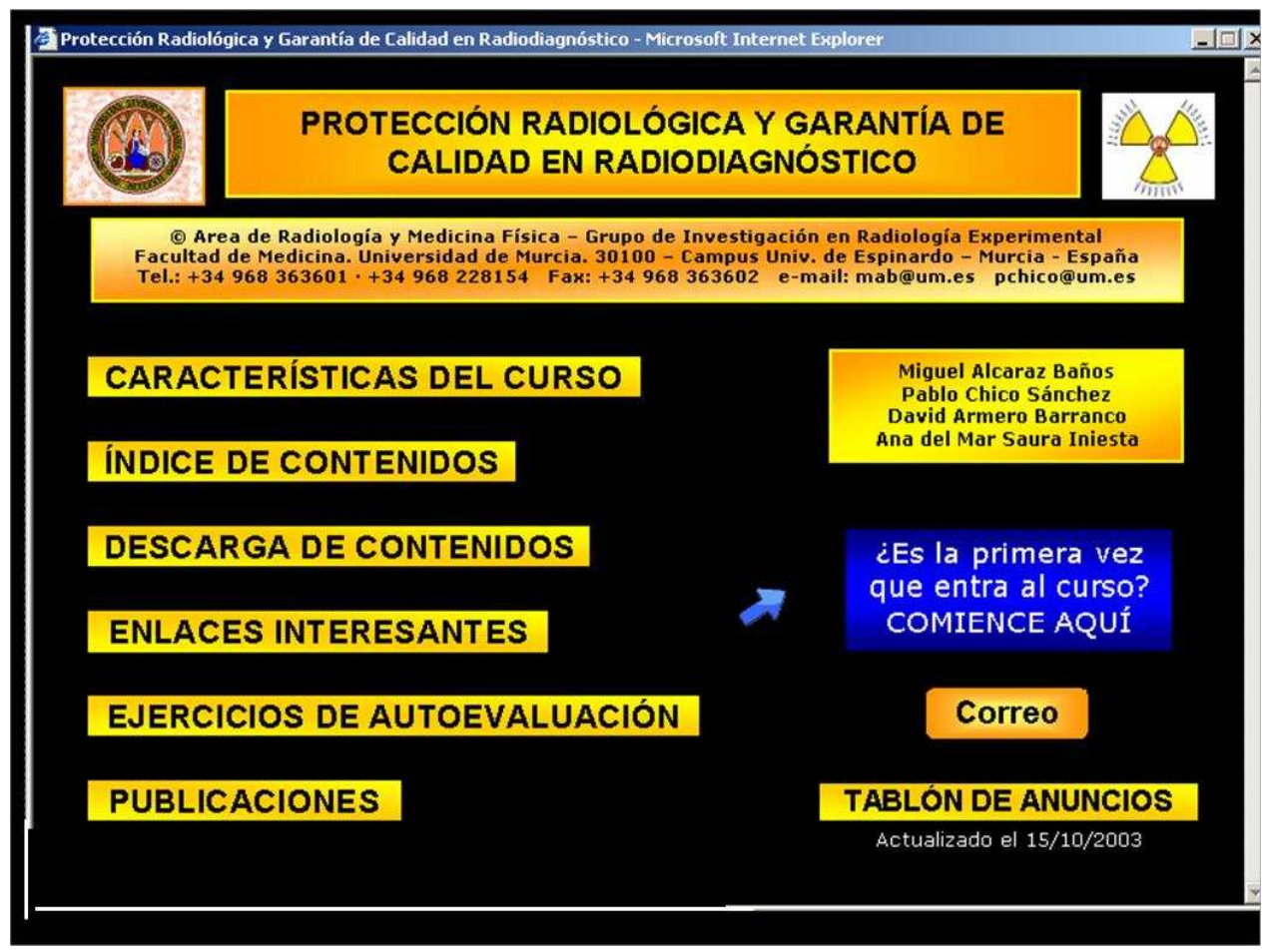

Fig.8: Página principal del curso en las primeras ediciones que te dirige a cada uno de los apartados del mismo y a los ejercicios de autoevaluación del alumno y evaluación del profesor.

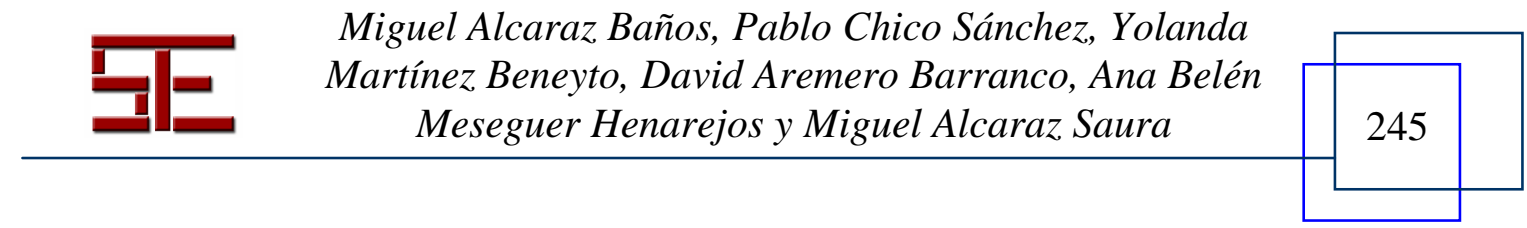



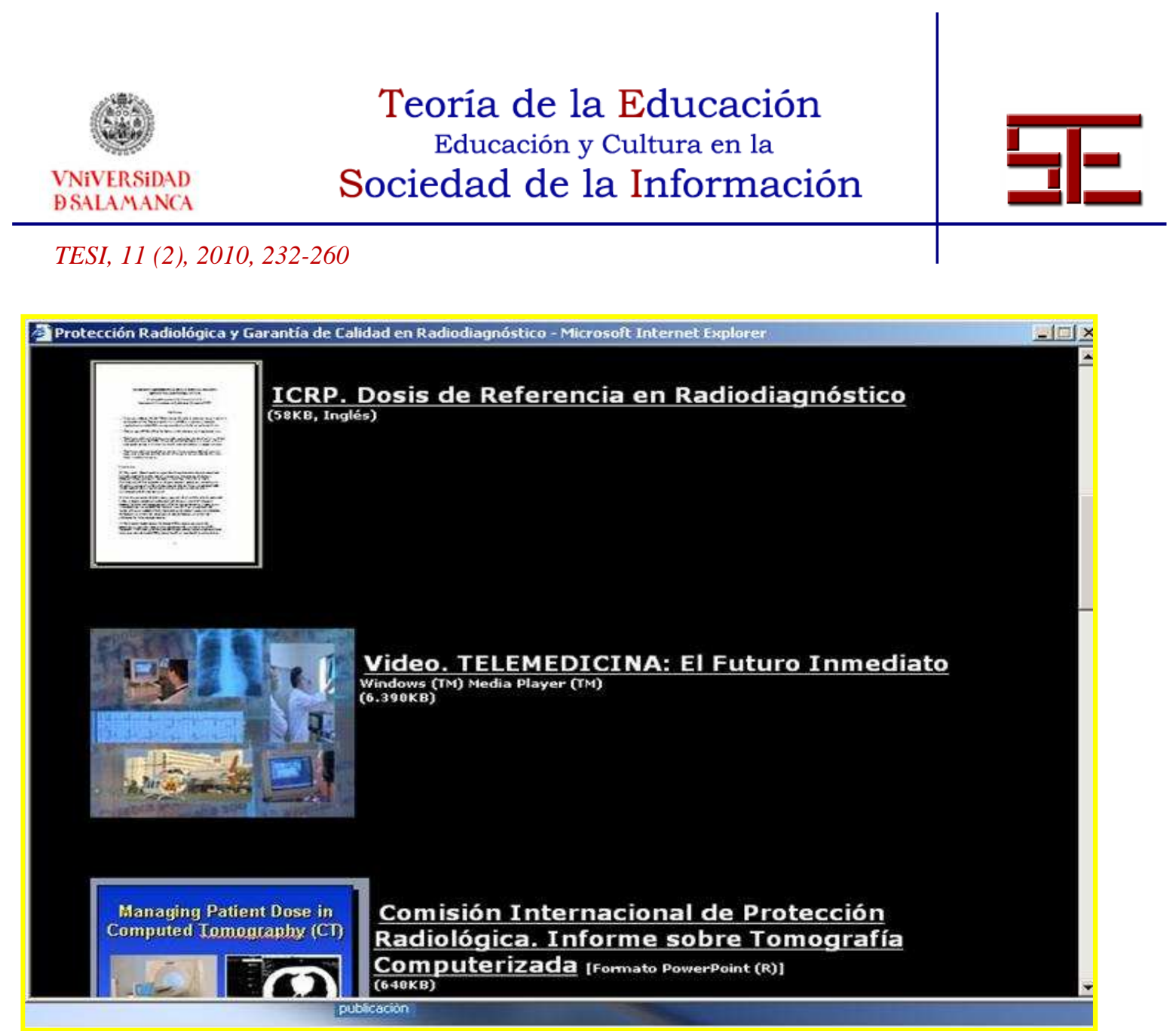

Fig.9: Página de publicaciones y recursos complementarios a los contenidos evaluables durante en el formato utilizado durante las 5 primeras ediciones.

Las actualizaciones y modificaciones en el desarrollo de las diferentes ediciones han sido importantes y motivadas fundamentalmente por el rápido desarrollo tecnológico y social. Así, en la primera edición realizada en el año 2000, únicamente el $40 \%$ de los alumnos decía tener un ordenador en casa y sólo el 5\% lo utilizaba para trabajar o estudiar. Pero, además, sólo el 30\% disponía de cuenta de correo electrónico y más del $76 \%$ de los alumnos consideraba que sus conocimientos informáticos eran mínimos, ya que sólo utilizaban el ordenador de forma ocasional. Era infrecuente tener una conexión a Internet y ésta generalmente era a través de línea telefónica. En este curso (año 2000) fue necesario asistir a los alumnos próximos (de la propia Universidad) con un apoyo presencial, fundamentalmente dirigido al manejo informático. Sin embargo, en estos momentos (2008-2009), el 92\% de los alumnos dispone de ordenador personal antes del comienzo del curso y casi todos (98\%) lo han utilizado para estudiar, aunque sea sólo de forma ocasional. Sólo el $15 \%$ de los alumnos reconoce conocimientos informáticos mínimos, pero coincide (15\%) con el número de alumnos que reconoce tener conocimientos avanzados e incluso de programación. El resto se reconoce como usuario frecuente y conocimientos suficientes para el manejo de los programas habituales. Sólo el

Miguel Alcaraz Baños, Pablo Chico Sánchez, Yolanda

$246 \quad \begin{gathered}\text { Martínez Beneyto, David Aremero Barranco, Ana Belén } \\ \text { Meseguer Henarejos y Miguel Alcaraz Saura }\end{gathered}$




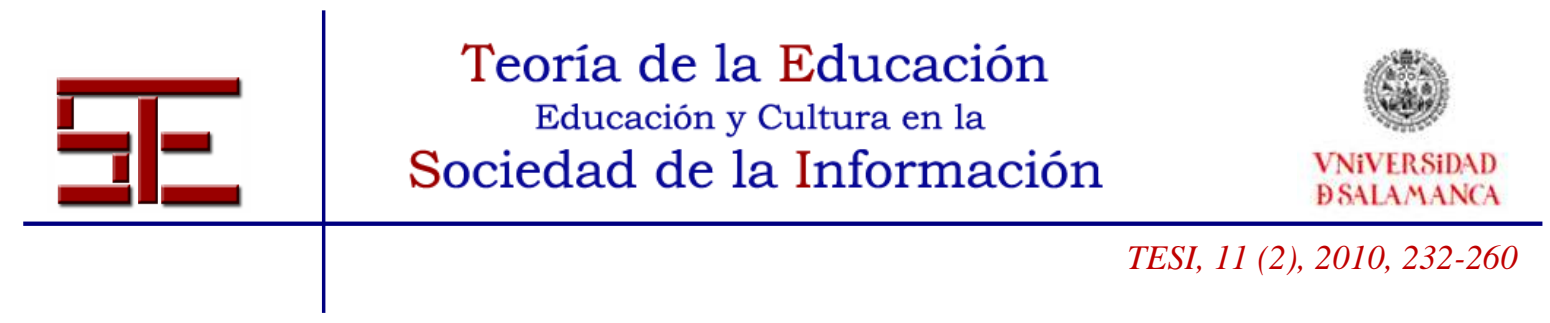

$2 \%$ no tiene cuenta de correo electrónico, aunque el $75 \%$ prefiere la utilización de cuentas de servidores gratuitos y no utilizan los servidores institucionales o universitarios.

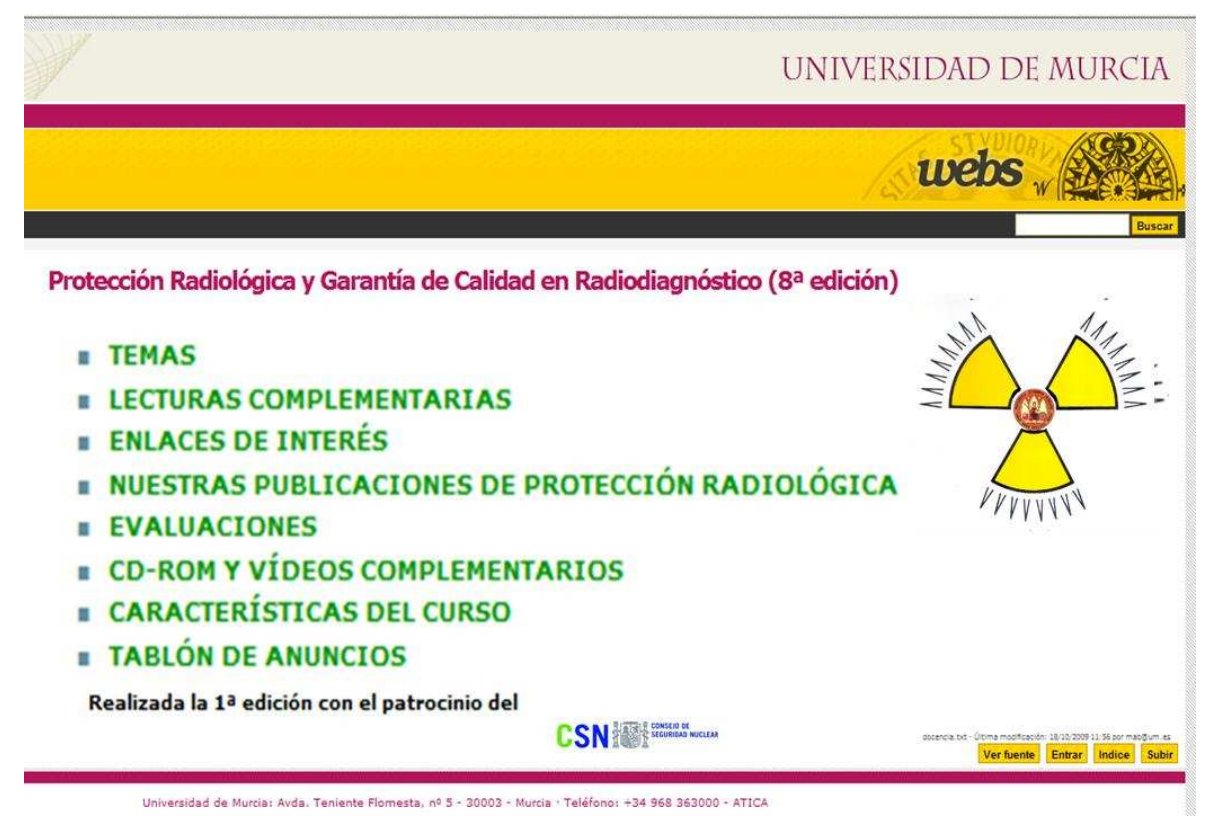

Fig.10: Página actual de entrada al Curso de tele-enseñaza que se encuentra ofertado con acceso libre en la página http://webs.um.es/mab/miwiki/doku.php?id=docencia de la Universidad de Murcia. Sus materiales y la mayoría de sus recursos están disponibles libremente, aunque las ediciones oficiales sólo se realizan durante los períodos aprobados por la Universidad de Murcia.

Un ejemplo del curso lo constituyen los resultados de la cuarta edición del mismo que correspondiente al curso académico 2004-2005. El curso fue seguido en su tercera edición, por un total de 222 estudiantes de los cuales 52 eran licenciados universitarios (médicos, odontólogos, físicos, químicos, biólogos e ingenieros), 12 diplomados universitarios (enfermeros y fisioterapeutas), 9 Técnicos Especialistas en Radiodiagnóstico y 6 auxiliares de enfermería. Pero además, junto con ellos, los alumnos de pregrado fueron 143 estudiantes que procedían de 10 diferentes países: 5 países europeos (España, Finlandia, Italia, Bélgica y Francia) y de 5 países Latinoamericanos (Argentina, Cuba y Colombia con 3 alumnos cada uno de ellos; México con 2 y Paraguay con 1 alumno). Los alumnos que siguieron el curso en España provenían de las provincias de Vizcaya, Palma de Mallorca, Badajoz, Albacete, Sevilla, Alicante, Madrid y Murcia. Estos alumnos españoles son estudiantes de Medicina, Odontología, Enfermería, Fisioterapia y Biología. Solamente 3 de todos los estudiantes, no fueron capaces de realizar con éxito los test de evaluación continua necesarios para obtener el diploma final del curso, fundamentalmente debido a su incapacidad para acceder a Internet y realizar el seguimiento del curso.

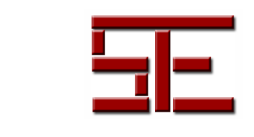

Miguel Alcaraz Baños, Pablo Chico Sánchez, Yolanda Martínez Beneyto, David Aremero Barranco, Ana Belén Meseguer Henarejos y Miguel Alcaraz Saura 


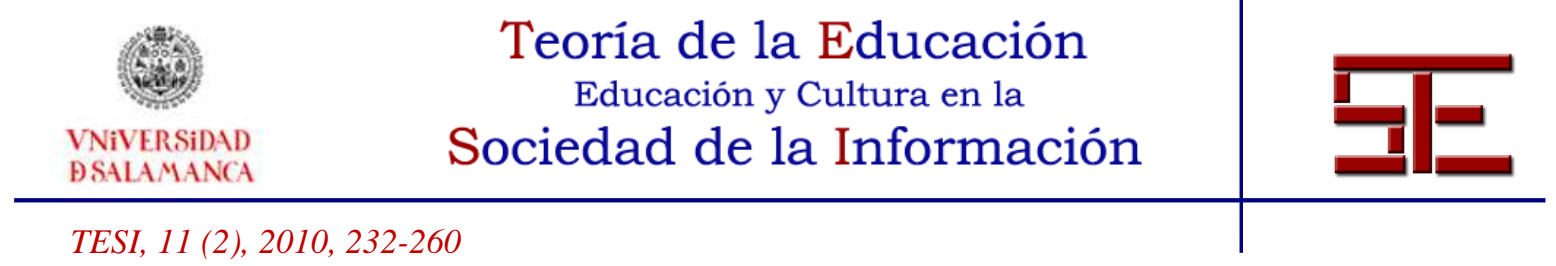

El análisis de las estadísticas de acceso al servidor reveló que los alumnos seguían el curso con mayor preferencia en la franja horaria entre las 18 y 19 horas (27\%), siendo el miércoles el día de la semana con mayor número de accesos $(30 \%)$. El total de visitas (entradas al servidor) durante la realización de esta edición ascendió a 12.002 entradas contabilizadas por nuestro propio servidor. De las estadísticas del servidor destaca que el acceso a los contenidos multimedia es aceptado por el alumnado, puesto que el número de descargas de los materiales en formato Windows Media (vídeos), fue similar a las descargas de archivos (pdf y Word) que contenían los temas teóricos evaluables directamente. Las estadísticas de error de acceso demostraron una buena fiabilidad del sistema puesto que los mensajes de error fueron en un $90 \%$ fallos del alumno al introducir la contraseña de acceso de entrada al sistema. La fiabilidad de la red y de la infraestructura de la Universidad de Murcia es aceptable, puesto que en ningún momento el servidor del curso estuvo inaccesible, salvo en las tareas propias de mantenimiento del mismo por parte de la Universidad de Murcia, que se hacían coincidir con las horas de menor acceso de los alumnos, según las muestras de tráfico obtenidas de las estadísticas de nuestro servidor.

Se realizaron 7 ejercicios parciales de evaluación y un ejercicio de evaluación final de los contenidos previamente definidos como "evaluables" y que se corresponden con la Guía Europea de Formación en Protección Radiológica (Figura 11), cuyos resultados fueron satisfactorios, superando los objetivos previamente establecidos. Al finalizar el curso, se invitó a los alumnos a que respondiesen a un cuestionario docente sobre diversos aspectos del mismo, en el que se preguntó sobre la facilidad de acceso y de los contenidos que mostraron unos resultados satisfactorios.

De las respuestas obtenidas en estos cuestionarios docentes se puede conocer que los alumnos consideraron que habían dedicado a este curso entre $2 \mathrm{~h}$ y $11 \mathrm{~h}$ semanas durantes las 14 semanas del mismo, con una media de 7 horas de estudio por semana. Los contenidos teóricos del curso, las explicaciones escritas, las publicaciones libres ofertadas y las imágenes y vídeos utilizados en el curso son, en un $85 \%$, buenas (nivel 4) o excelentes (nivel 5) en una valoración del 1 al 5. La utilidad de los enlaces expuestos se ha valorado como excelente (nivel 5) en un $80 \%$ y la utilidad de los bloques o actividades prácticas ha sido mayoritariamente considerada sólo de aceptable (nivel 3).

Para el $97 \%$ de los alumnos, ha sido el primer curso realizado "on-line" a través de Internet, destacando fundamentalmente la libertad de horarios, facilidad de acceso y comodidad de seguimiento. Las críticas más frecuentes $(15 \%)$ se han dirigido a la necesidad de disponer de un ordenador, con conexión a Internet (12\%), sobre todo, en estudiantes fuera de su domicilio habitual; así como a los largos periodos de mantenimiento interno de las Red universitaria que imposibilitaba el acceso a los servidores del curso (5\%). El 90\% de los alumnos describe su experiencia en la realización del curso como

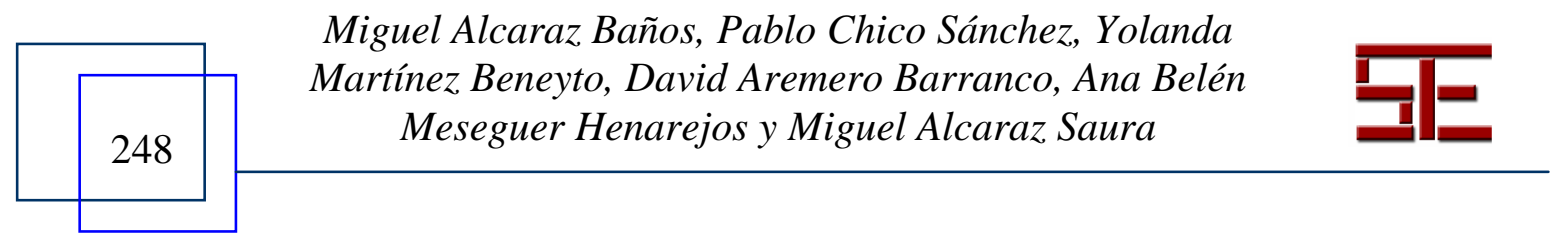




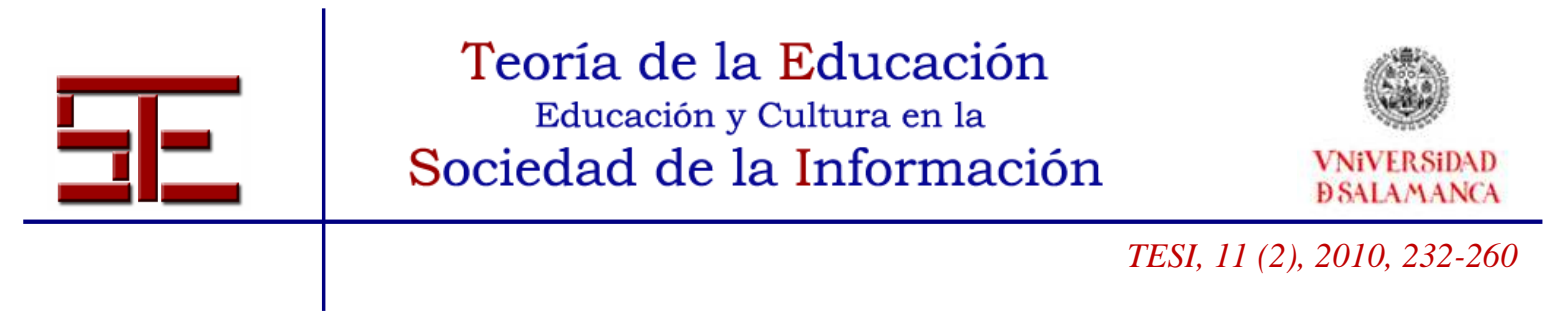

útil, innovadora y necesaria. Consideran que la incorporación a estos sistemas de educación va a ser importante en su desarrollo profesional próximo. Sin embargo, casi el 10\% de los alumnos se muestra claramente en contra del procedimiento de enseñanza aprendizaje por diferentes motivos: necesidad de estudiar sólo o aislado a pesar de los foros y chats disponibles, aversión al uso del ordenador, dificultades de conexión a Internet, ausencia de clase magistral presencial impartida por el profesor, situaciones de relación impersonal y poco motivadora para la actividad del alumno. En casi el $1 \%$ de estos alumnos el rechazo a este sistema de enseñanza es completo.

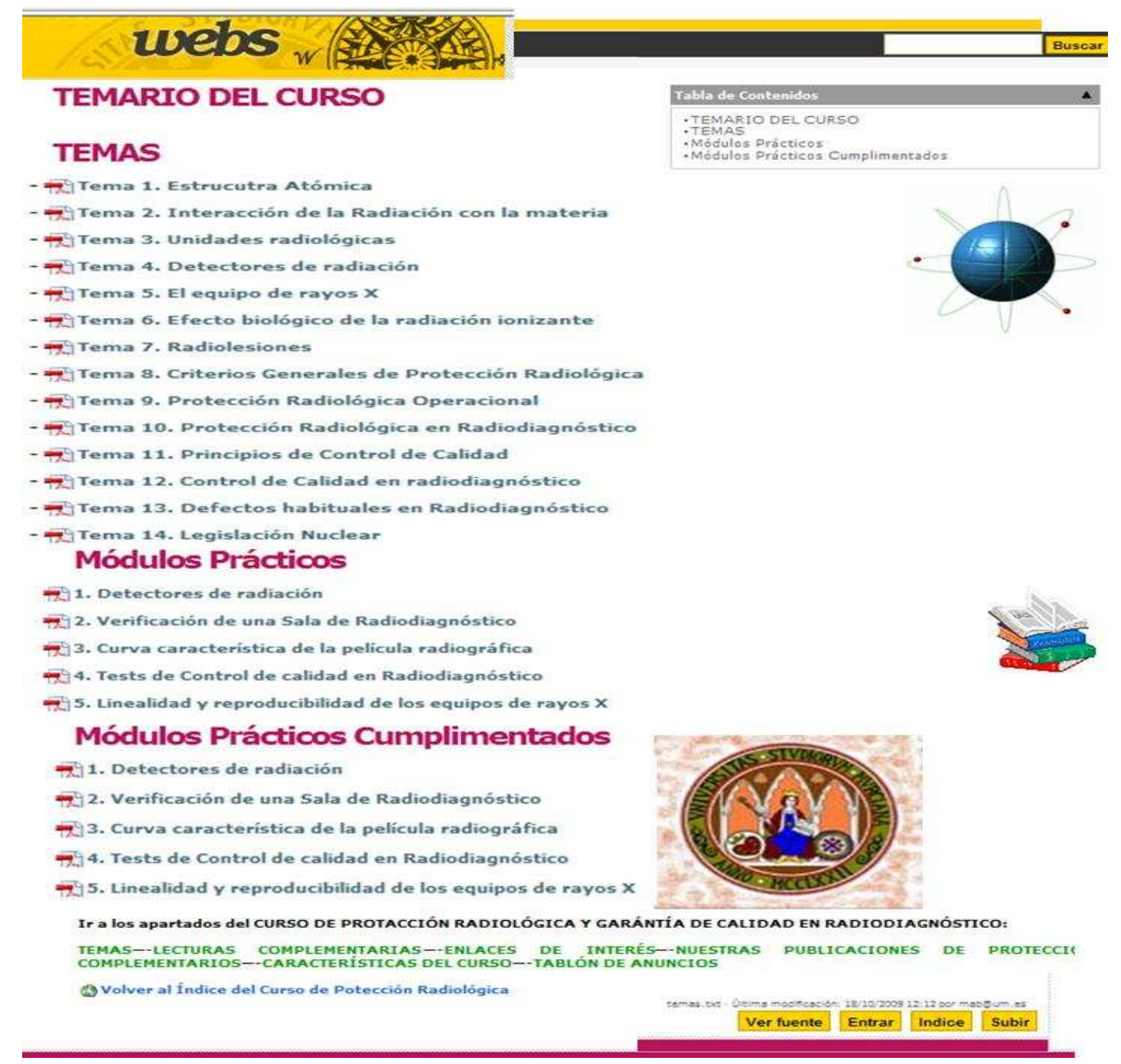

Fig.11: Contenidos evaluables del curso en correspondencia con las "Guidelines on Education and training in radiation protection for medical exposures. Radiation Protection $n^{\circ} 116$ " (European Commission 2000).

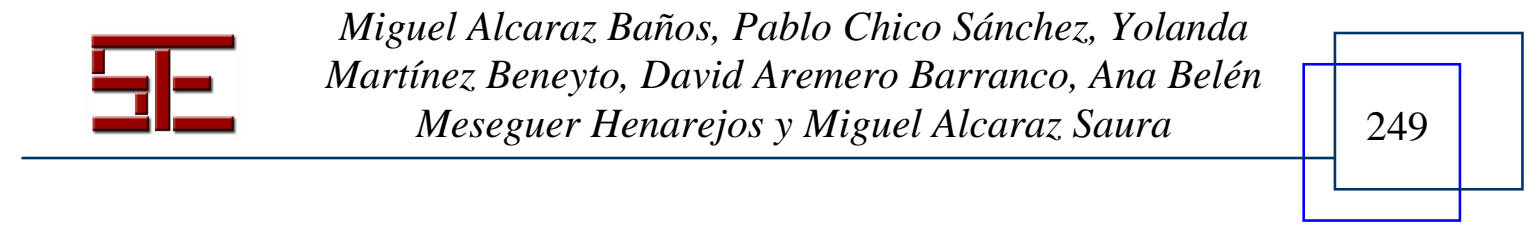




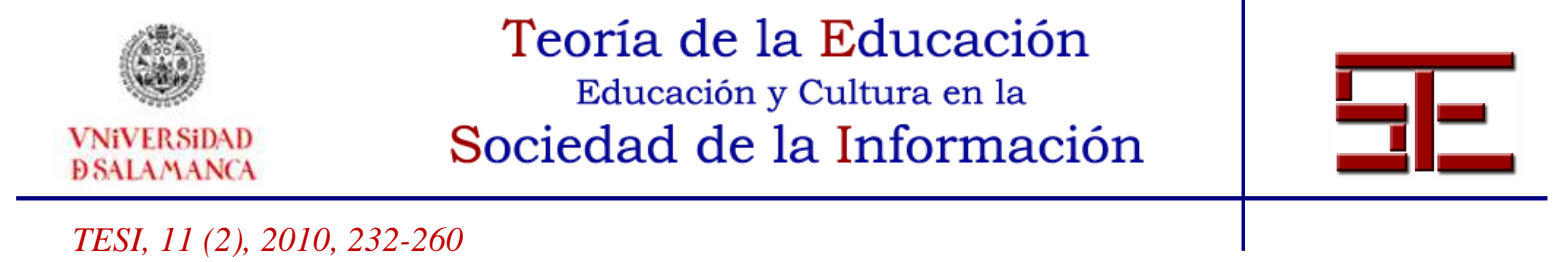

Ante la solicitud de propuestas de desarrollo, la mayoría de los alumnos, agradeciendo la calidad de los materiales y recursos presentados, suelen demandar mayor cantidad de vídeos especializados en línea con los ya confeccionados y aumento de programas informáticos y CD-Roms interactivos e imágenes que complementen los contenidos ofertados. Solicitan también el incremento de foros o chats entre grupos de alumnos que puedan permitir un trabajo interactivo en grupos pequeños y que complemente el único chat habilitado, hasta el momento, para los alumnos del curso.

El 90\% de los alumnos de la Universidad de Murcia considera que este curso es una excelente idea para la obtención de los Créditos de Libre Configuración por Equivalencia y útil para la preparación de las asignaturas del área de Radiología y Medicina Física. La inclusión de un número de preguntas en los ejercicios finales de evaluación en la Asignatura de Radiología y Medicina Física y su comparación con los datos de otros años académicos en los que los alumnos no habían realizado el curso de tele-enseñanza reveló un aumento de las calificaciones finales obtenidas por aquellos alumnos que siguieron el desarrollo del curso (15\%), sin obtener diferencias estadísticamente significativas.

Sin embargo, los cuestionarios docentes realizados al grupo de profesores del curso muestran una situación diferente y que muestran las tres dificultades más importantes en el desarrollo de este tipo de enseñanza. En primer lugar, la necesidad de realizar la preparación de los materiales y recursos audiovisuales originales o de libre acceso con antelación a la realización de estos cursos, y que deben de estar presentados (textos, vídeos, presentaciones y programas informáticos) como recursos digitalizados en los medios informáticos disponibles; situación agravada por la escasez medios didácticos de libre acceso existentes y una legislación muy restrictiva que prohíbe la difusión de dichos materiales a través de Internet (100\%). En segundo lugar, el tiempo necesario para que el profesor pueda adquirir los conocimientos informáticos suficientes para el desarrollo del curso y que, además, le permita atender a los alumnos de forma adecuada; que se suma, en un curso de 14 semanas al tiempo necesario para mantener las conexiones sincrónicas y asincrónicas mediante tutorías individualizadas que exigen una enorme cantidad de tiempo de profesor, todo ello, perdiendo la relación personal con el alumno (100\%). En tercer lugar, la totalidad del profesorado también manifiesta la escasa valoración institucional por las autoridades académicas universitarias de este tipo de actividades que no se tienen en cuenta en la promoción del profesorado universitario. Por ello, se involucran en este proyecto en la creencia de que el sistema permite una mejoría evidente frente al sistema tradicional pero que se realiza exclusivamente por interés personal y basado en la voluntariedad de participar en este tipo de proyecto.

Según la encuesta docente, es opinión mayoritaria de los profesores que las actividades prácticas de tipo colaborativo y base multimedia (presentaciones PowerPoint, comenta-

Miguel Alcaraz Baños, Pablo Chico Sánchez, Yolanda Martínez Beneyto, David Aremero Barranco, Ana Belén Meseguer Henarejos y Miguel Alcaraz Saura

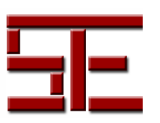




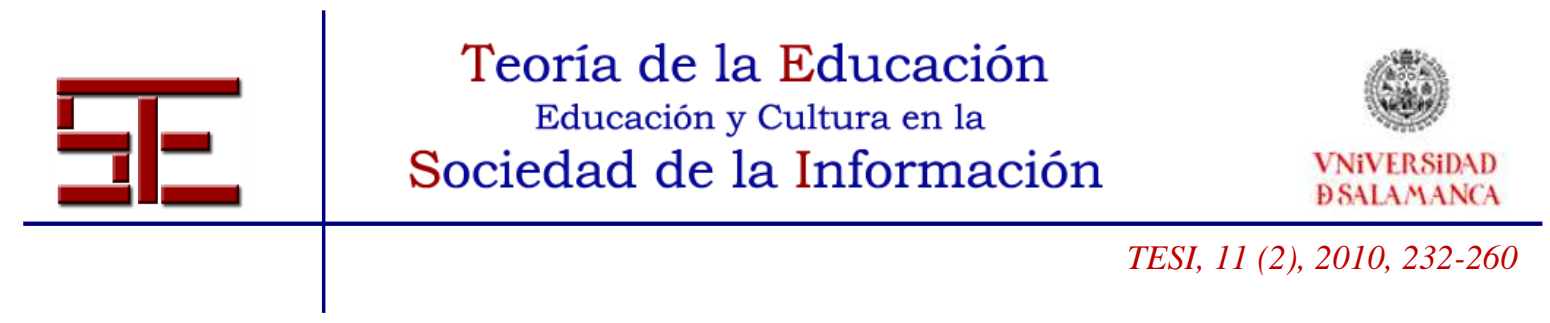

rios de artículos colaborativos con Word o pdf, incluso algún vídeo con programas digitales...) son bien aceptadas y bien realizadas por el alumnado. Sin embargo, las actividades prácticas especializadas (tests de control de calidad, interpretación de imágenes radiológicas, defectos y errores técnicos más frecuentes) de importancia en la formación y capacitación profesional, no se alcanzan adecuadamente con este tipo de formación y se consideran insuficientes para obtener un adecuado aprendizaje de las técnicas y habilidades prácticas. Parece necesario exigir un contacto más directo, de tipo presencial, para confirmar la obtención de la suficiente capacitación profesional.

Todas las ediciones han sido aprobadas oficialmente como créditos por equivalencia dentro de los Créditos de libre configuración todos los alumnos que cursen las Licenciaturas de Medicina (9 créditos) y Odontología (4'5 créditos), así como las Diplomaturas de Fisioterapia (4 5 créditos) y Enfermería (2 créditos), según las normativas específicas de cada centro en la Universidad de Murcia. Al finalizar el curso, y habiendo superado la calificación mínima acordada por los docentes, al alumno se le hacía entrega de un diploma acreditativo, avalado por la Universidad de Murcia (Curso de Promoción Educativa), por un total de 115 horas (Figura 12). Sobre el desarrollo de las diferentes ediciones del curso se han presentado algunas comunicaciones a Congresos y reuniones de carácter internacional, analizando diferentes aspectos de las posibilidades de la teleenseñanza a través de Internet tanto durante los estudios de pregrado (Alcaraz et al., 2002a, 2002b), como para el postgrado y cursos de formación continuada (Alcaraz et al., 2003a, 2003b, 2004a, 2006b).

\section{4.- DISCUSIÓN}

En la literatura revisada se han podido encontrar dos posturas claramente definidas y diametralmente opuestas: aquellos que defienden el uso del sistema tradicional por dar buenos resultados y descartar cualquier otra posibilidad; y aquellos que con la aplicación de las nuevas tecnologías han obtenido tan buenos resultados como los primeros (Torales, 2008; Sánchez-Llorente, 2009).

Hace tres décadas que se publicaron los primeros artículos reclamando mejoras en la formación radiológica de los estudiantes (Squire y Becker, 1975). Aunque se han realizado avances significativos durante este tiempo, la educación radiológica en las diferentes carreras universitarias se han mantenido considerablemente por detrás de los acontecimientos (Subramaniam y Gibson, 2007). De hecho se continúan publicando en nuestros días similares reclamaciones para mejorar la enseñanza de los diferentes aspectos de la radiología (Holt, 2001). Nosotros coincidimos con Torales (2008) en que una de las mayores deficiencias en la educación en Radiología, y en nuestra Universidad en general, es la falta de reconocimiento de la enseñanza por las diferentes entidades públicas. En los hospitales, se sigue muy de cerca los resultados clínicos y de investigación,

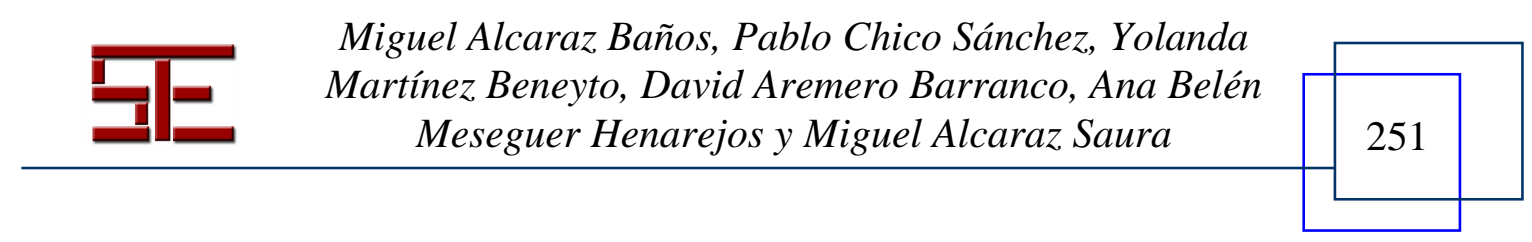




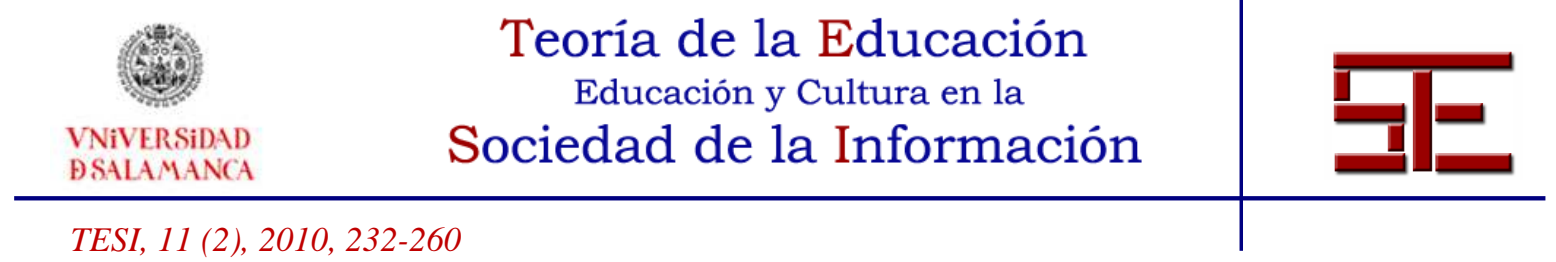

pero en mucha menor medida los resultados educativos. Nosotros nos atrevemos a añadir que ocurre exactamente lo mismo que en nuestra Universidad.

Una parte considerable de los proyectos de enseñanza asistida por ordenador son del ámbito de la Radiología (Torales, 2008). Letterie (2003) realizó una revisión de los trabajos publicados sobre enseñanza asistida por ordenador en Medicina entre 1988 y 2000, encontrando 210 estudios que incluían tecnología diversa "on-line", Cd-Roms, Disco Vídeo Láser, estaciones de trabajo multimedia, realidad virtual y simulación. El $24 \%$ de estos estudios eran del campo de la radiología, sólo detrás de los que se incluían en el campo de la medicina interna y que alcanzaban el $34 \%$ de todos los estudios analizados. En todos estos artículos se recoge que, en una descripción inicial, la enseñanza asistida por ordenador es innovadora, prometedora y entusiasta. Sin embargo, hasta hace sólo unos años todavía se mantenía que eran necesarios futuros estudios que no solamente probasen la superioridad de esta tecnología, sino que demostrasen de forma concluyente que no producen detrimento en la educación profesional (Squire y Becker, 1975; Friedman, 1994). Esta es la situación inicial que nos impulso, en aquel momento, a introducirnos en este tipo de enseñanza.

Después de 12 años de trabajo en la formación asistida por ordenador y de la realización de cursos a través de Internet nos sumamos, junto con nuestros alumnos a las ventajas de estas nuevas herramientas educativas, especialmente las que caracterizan la formación a través de Internet (Alcaraz et al., 2003a, 2003b, 2004), y que han descrito numerosos autores: interactividad, multimedia, sistema abierto, independencia del espacio-tiempo, publicación electrónica, acceso a recursos ilimitados, comunicación intercultural, multiplicidad de expertos, control del alumno sobre su propio aprendizaje, ausencia de discriminación, aprendizaje colaborativo, coste razonable y evaluación "on-line" de alumnos y profesores (Añel, 2009; Sánchez-Llorente, 2009).

Numerosos autores consideran que el em-

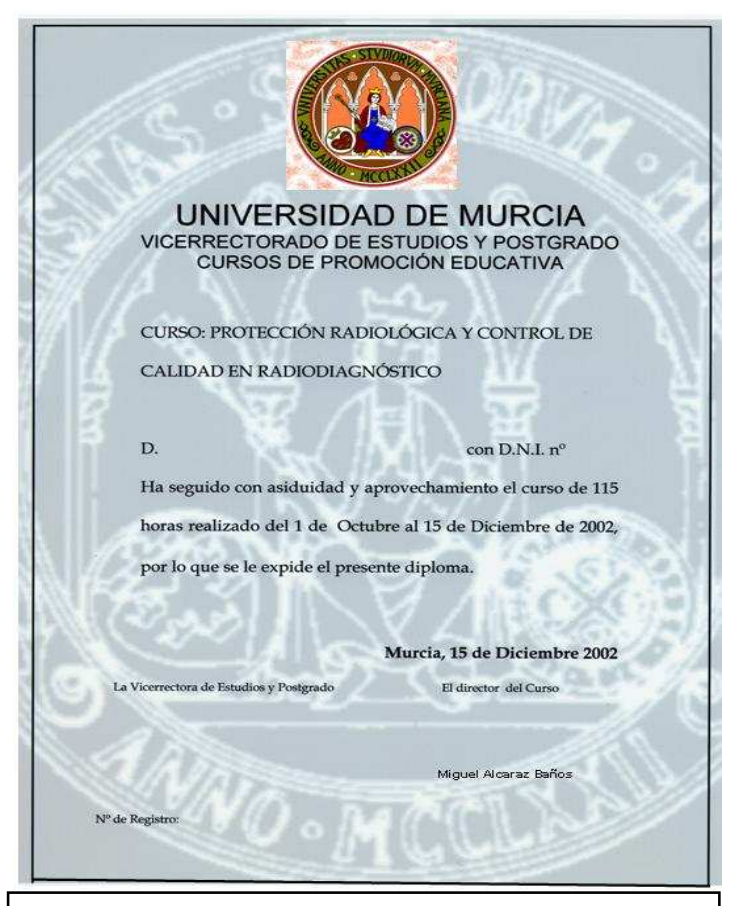

Fig.12: Diploma oficial acreditativo de el aprovechamiento del curso de tele-enseñanza.

pleo de sistemas multimedia y de la enseñanza asistida por ordenador es más eficaz para el aprendizaje que el método de enseñanza tradicional y produce una mejora evidente sobre el rendimiento de los alumnos (Goldman y Blake, 1996; Rouse, 1999; Pelayo-

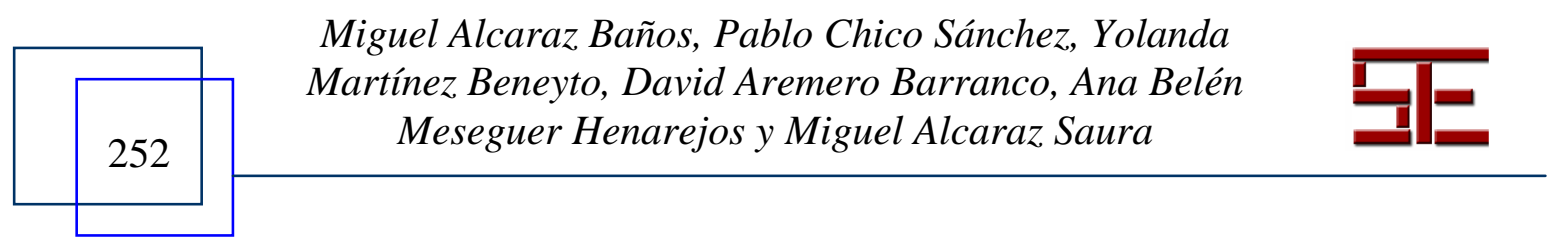




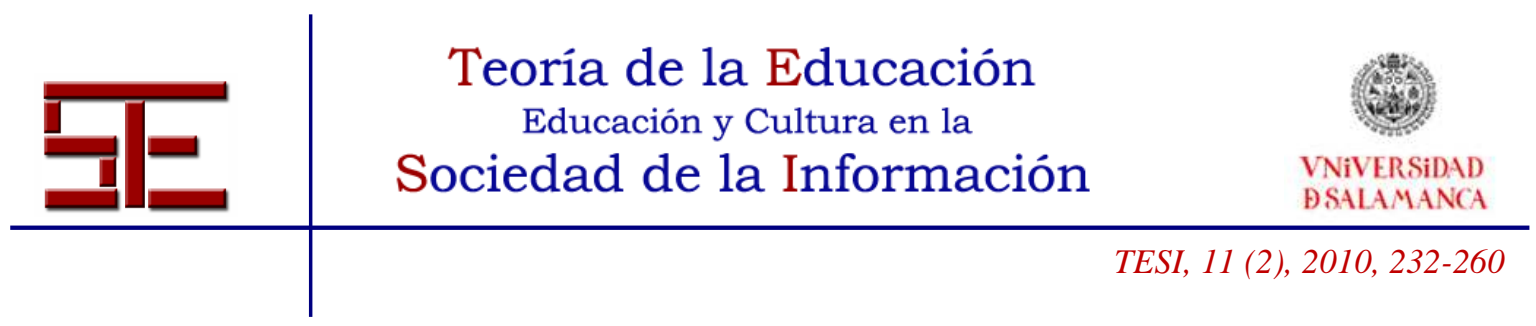

Alvarez et al., 2000; Fleetwood et al., 2002; Alcaraz, 2006b;). Incluso, algunos autores afirman que cuanto más multimedia e interactivo sea el método didáctico mayor será la participación del estudiante y mayor su efectividad en el aprendizaje (Goldman y Blake, 1996). En nuestra experiencia hemos podido demostrar un mayor interés de los alumnos por los temas tratados, un mayor cumplimiento de las tareas encomendadas y una mayor demanda de tareas de orientación hacia el profesor (Alcaraz et al., 2002a, 2002b).

Nosotros podríamos estar completamente de acuerdo con todas las ventajas descritas anteriormente sobre este sistema de enseñanza, si nuestros resultados sobre las actividades prácticas específicas y de capacitación profesional hubieran conseguido alcanzar el mismo nivel y los objetivos propuestos que hemos obtenido con los contenidos meramente teóricos o de divulgación e instrucción. Sin embargo, eso no ha sido así. La interacción con el paciente, el aprendizaje de habilidades prácticas necesarias para el desarrollo profesional real en materias de las Ciencias de la Salud, no puede permitir dejar sin control la adopción de medidas correctas que pueden tener incidencia directa sobre la salud o protección de las personas. Por ello, coincidiendo con otros autores, la enseñanza asistida por ordenador no debe sustituir completamente el método tradicional cara a cara (profesor-estudiante). Ciertos aspectos, como las habilidades prácticas ("saber hacer") deben de aprenderse en el contexto de la práctica en el mundo real, lo que también puede verse favorecido con la utilización de las tecnologías que la emulen. Para algunos autores, las formas adecuadas de interaccionar con colegas y pacientes no pueden memorizarse en la pantalla de un ordenador (Lieberman, 2002; Torales, 2008). Pero además, y también en nuestro caso, una limitación crucial de la enseñanza asistida por ordenador es que no se puede reemplazar la interacción cara a cara con el estudiante, pues se priva a ambos de una de las mayores recompensas del proceso de enseñanzaaprendizaje. Situación que no ocurre cuando se propone, como también es nuestro caso, que esta enseñanza sea un complemento a la interacción profesor-alumno (Torales, 2008).

En nuestro medio, el mayor problema de la utilización docente de las nuevas tecnologías se encuentra en conseguir que el docente, especialista en su materia, no sólo cree los textos, medios y recursos especializados (Alcaraz et al., 2002a, 2002b); sino que también domine dichas técnicas con suficiente nivel como para poder incorporarlas adecuadamente a este tipo de enseñanza-aprendizaje. Ya en 1991 se describía que el coste de desarrollar los sistemas de enseñanza asistida por ordenador incluye el coste de tiempo de profesor. Se ha descrito que, incluso cuando el docente tiene suficiente nivel de experiencia con la tecnológica informática, el desarrollo de programas asistidos por ordenador requiere tanta cantidad de tiempo y energía que, al menos, es equivalente a la de escribir un libro (Keane et al., 1991).

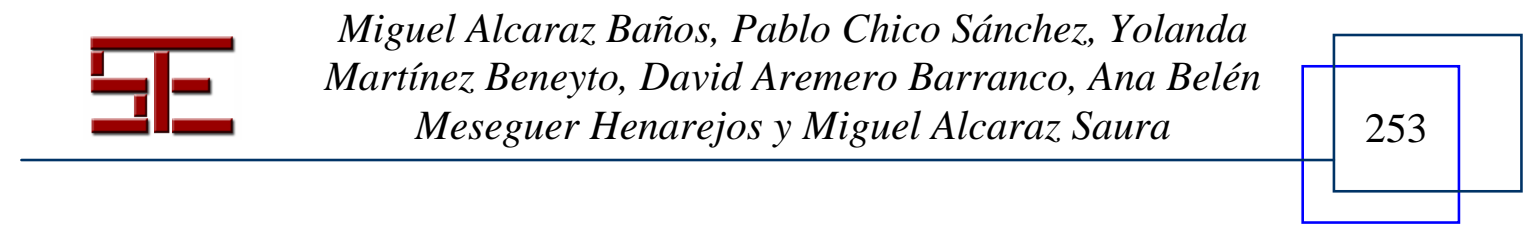




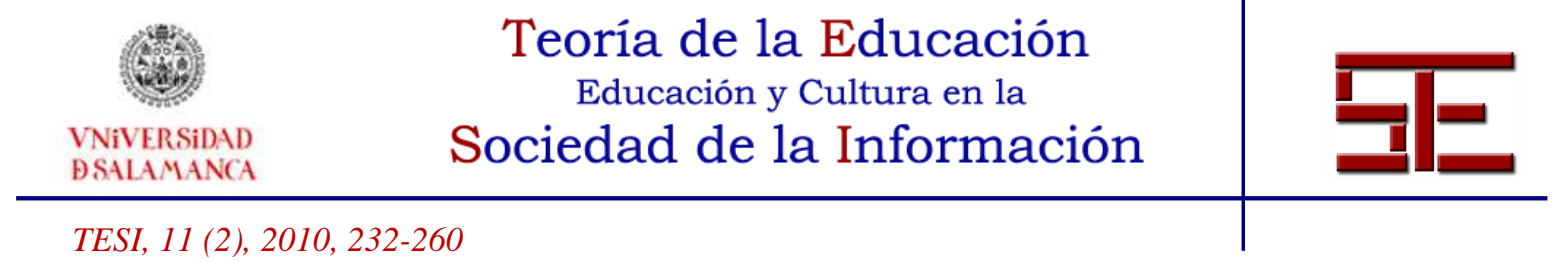

Nuestro curso se ha desarrollado sobre una línea prioritaria de la Unión Europea (radioprotección), intentando establecer redes de comunicación entre los diferentes estamentos que trabajan con la radiación ionizante. Por ello, se han obtenido diferentes ayudas de Instituciones Públicas (Consejo de Seguridad Nuclear, MEC, CARM) que han permitido la utilización de las nuevas tecnologías en la enseñanza de nuestro campo de interés (Alcaraz et al., 2003a, 2004a). Desde que comenzamos, supimos que no sería valorado institucionalmente de forma adecuada en nuestra carrera universitaria como cualquier otro trabajo de investigación, ya que se carece de cualquier tipo de Factor de Impacto docente; una gran diferencia respecto al Factor de Impacto de las revistas científicas especializadas que son sistemáticamente aplicadas en la evaluación del profesorado en las Ciencias de la Salud. En realidad, es una clara correspondencia con lo que ocurre en nuestro medio universitario local: es la diferencia entre los "escalones" de investigación frente a los "escalones" de actividad docente, en el desarrollo profesional del profesor universitario.

En nuestro estudio sólo el $10 \%$ de los alumnos se muestra disconforme con este sistema de enseñanza, y otro $1 \%$ de los estudiantes muestra un rechazo frontal a este tipo de enseñanza, lo que coincide con los autores que afirman que el uso alternativo de esta metodología tecnológica es aceptado favorablemente por la mayoría de los estudiantes para su formación. Pero en la bibliografía revisada sólo se insinúa ligeramente la denuncia de nuestros profesores sobre la escasa valoración del esfuerzo y dedicación que han de realizar los profesores y del enorme tiempo que han de prestar a este tipo de enseñanza, sobre todo debiendo mantener los niveles científicos exigidos para su promoción personal, en donde se valoran otros méritos de investigación casi de forma exclusiva (Torales, 2008; Sánchez-Campillo, 2009).

El reto actual lo constituye nuestra adaptación a la Enseñanza Superior en el Espacio Europeo ya que con su llegada, la redefinición de la concepción de crédito, que incluye las horas de estudio, seminarios, etc., que el alumno desarrolla, y la progresiva desaparición de las clases magistrales para ser reemplazadas por tutorías, cursos y seminarios, hacen que la realización de cursos de capacitación, tele-enseñanza, y formación continuada, sean un elemento importante a tener en cuenta en la formación del futuro graduado y posgraduado universitario. La experiencia desarrollada por nuestro Grupo de Investigación en la labor de la tele-enseñanza, y los resultados obtenidos tras los cursos realizados, sugieren que el nivel de conocimientos adquiridos por nuestros alumnos, a través de este tipo de cursos, es similar a la formación recibida de forma tradicional, y en consecuencia, puede ser una de las herramientas básicas en el futuro proceso de enseñanza-aprendizaje dentro de las directivas en la implantación del Espacio Europeo de Enseñanza Superior en Ciencias de la Salud.

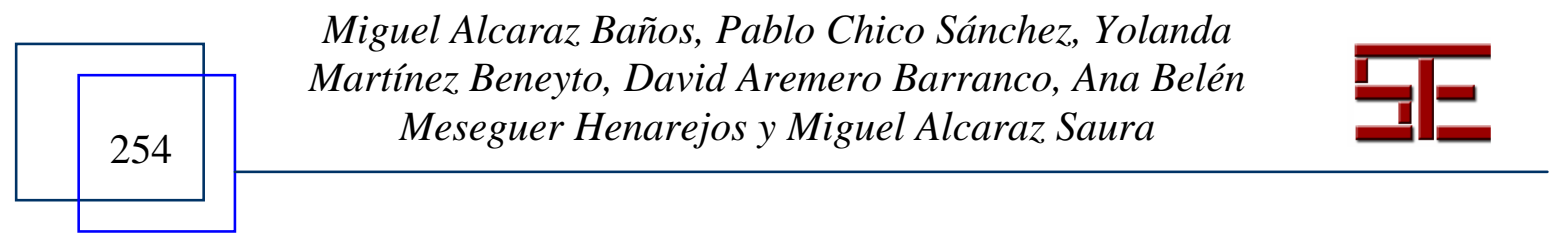




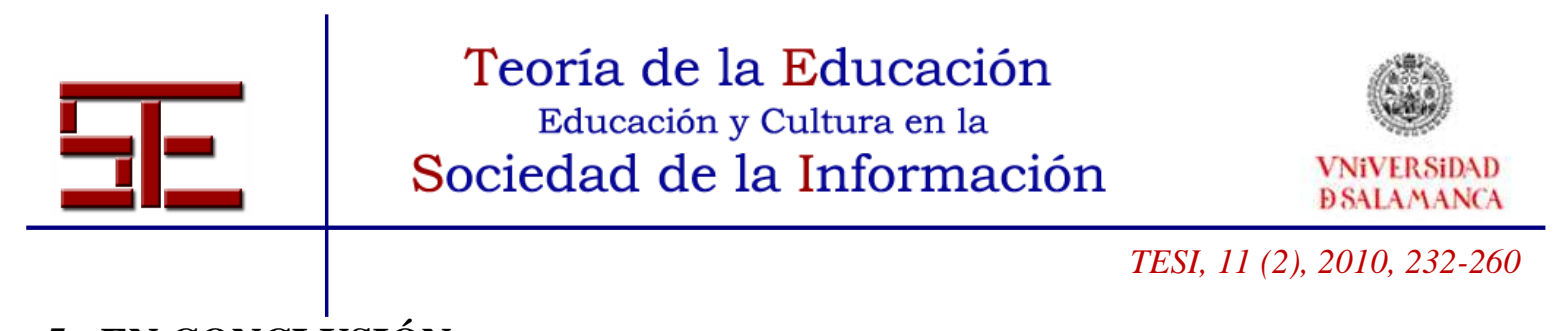

\section{5.- EN CONCLUSIÓN:}

- la utilización de material didáctico adecuado determina el nivel de conocimientos que puede llegar adquirirse, y por ello, posiblemente, la capacidad profesional inicial de los alumnos. El esfuerzo para generar nuevos materiales didácticos han de realizarlo las instituciones docentes, incentivando al profesor.

- El aprendizaje a través de Internet constituye una forma de enseñanza que utiliza las nuevas tecnologías para permitir el acceso a la educación desde cualquier lugar. Además, en estos momentos es una forma asequible y adecuada para analizar las imágenes diagnósticas en un soporte económico que es una demanda permanente de nuestros alumnos.

- Una vez finalizado el proceso de formación tanto universitaria como no universitaria, la tele-enseñanza permite un proceso de formación continuada donde es posible alcanzar objetivos básicos y especializados de Protección Radiológica y de Programas de Control de Calidad.

- Sin embargo, tras 12 años de desarrollo y defensa de la tele-enseñanza a través de Internet como procedimiento innovador de formación, consideramos que para el aprendizaje de aspectos y habilidades prácticas especializadas ("saber hacer") resulta insuficiente. En estos casos, junto a la divulgación de la información realizada mediante la tele-enseñanza y procedimientos digitales de alta calidad, se debería complementar con actividades prácticas presenciales que permitan adquirir dichas habilidades de una forma controlada y objetivada por el profesor, sobre todo en aquellas actividades que impliquen riesgos importantes a terceras personas por insuficiente habilidad técnica, como ocurre en el campo de las Ciencias de la Salud.

- Todo esto implica un esfuerzo enorme del profesor que no es valorado adecuadamente en su carrera profesional por los diferentes organismos que controlan y acreditan la promoción de las personas involucradas en la carrera universitaria. En estos momentos existe una enorme discrepancia entre lo que se debe hacer de forma obligatoria dentro del Espacio Europeo de Enseñanza Superior y los medios imprescindibles para acometer dicha tarea.

\section{6.- AGRADECIMIENTOS}

Este trabajo se ha realizado gracias a 6 ayudas y contratos públicos competitivos de investigación obtenidos del Ministerio de Educación y Ciencia (B.O.E. del 23/10/1997), de los programas I $+\mathrm{D}$ del Consejo de Seguridad Nuclear (MMC/SUBV/UMU/064/2003; CSN/GTP/RRII/SUB/63; CSN 24/09/2007,BOE n 106, 05/03/2007) y de la Comunidad Autónoma de la Región de Murcia en cofinanciación con los fondos FEDER de la Unión Europea (CARM 2S118 nº 200500067774 de 19/0/2006 y un Contrato de Investigación de la Universidad de Murcia, UMU-9094) .

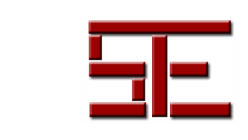

Miguel Alcaraz Baños, Pablo Chico Sánchez, Yolanda Martínez Beneyto, David Aremero Barranco, Ana Belén Meseguer Henarejos y Miguel Alcaraz Saura 


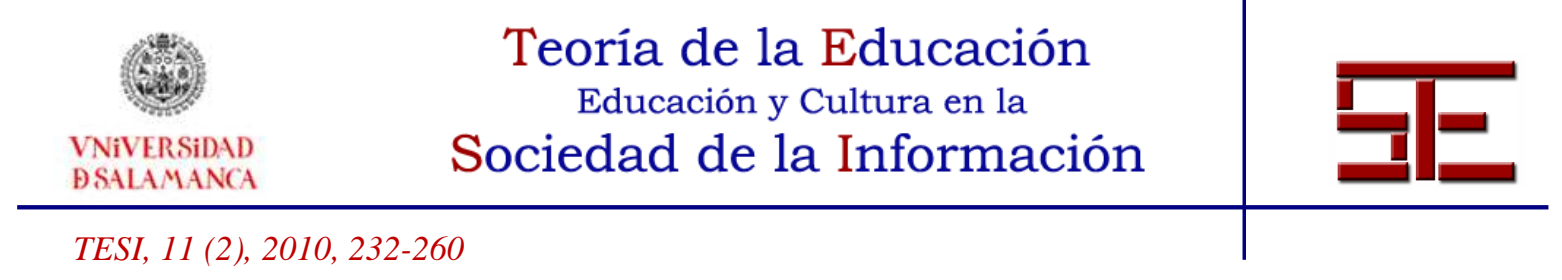

\section{7.- BIBLIOGRAFÍA}

Alacaraz, M. Y Genovés, J. L. (1996). Protección Radiológica en Radiodiagnóstico. Barcelona: Diego Marín (ICE-Universidad de Murcia).

Alcaraz, M., Jodar, S., Martinez Beneyto, Velasco, E. Y Chiva, F. (2000). La radiología panorámica en el radiodiagnóstico dental. Revista Europea de OdontoEstomatología, 12 (5), 263-270.

Alcaraz, M., Chico, P., Armero, D. Y Saura Iniesta, A. M. (2002a). Protección radiológica médica: Curso de Tele-enseñanza para la educación médica continuada. Informédica 2002: preparando el camino para la e-Salud Global. II Congreso Virtual iberoamericano de informática médica. Nov 4-30 en Internet.

Alcaraz, M., Chico, P., Saura Iniesta, A. M. Y Cabero, J. (2002b). Elaboración de material didáctico de Protección Radiológica y Control de Calidad en Radiodiagnóstico para el alumnado de Técnico Superior en Imagen para el Diagnóstico. $1^{\mathrm{a}}$ Jornada Universitaria sobre Multimedia y Tele-enseñanza en Radiología. Málaga, 31-Mayo2002, Libro de Resúmenes pp.: 65-66.

Alcaraz, M., Chico, P., Armero, D., Saura Iniesta, A.M. (2003a). Radiological protection and quality assurance in health sciences: Tele-education for continued postgraduate training. II International Conference: Radiation protection training. Future Strategies Madrid. 17-19 Septiembre. Procedings pp 263-267.

Alcarraz, M. (2003). Bases físicas y biológicas del radiodiagnóstico médico (2a edición). Murcia: Servicio de Publicaciones de la Universidad de Murcia.

Alcaraz, M., Chico, P., Armero, D., Saura, A. M. Y Vicente, V. (2003b). Protección radiológica y Garantía de calidad en ciencias de la salud: La tele-enseñanza para cursos de formación continuada. XVI Congreso Nacional de la Sociedad Española de Educación Médica. Madrid, Educación Médica, 5 (83), 47.

Alcaraz, M., Chico, P., Saura Iniesta, A.M., Armero, D. Y Vicente, V. (2004a). Course on radiological protection and quality assurance in radiology. Tele-education course: a possible solution to continued postgraduate training. XI International congress of the International Radiation Protection Association (IRPA 11). Madrid, 2328 May, pp.: 83-

Alcaraz, M., Martínez Beneyto, Y., Pérez, L., Jódar, S., Velasco, E. Y Canteras, M. (2004b). The status of Spain's dental practices following the European Union directive concerning radiological installations, Oral Surg Oral Med Oral Pathol Oral Radiol Endod, 98, 76-482.

Alcaraz, M., Martínez Beneyto, Y., Jodar, S., Velasco, E Y García-Vera, M.C. (2004c). Control de calidad en Radiología dental intraoral: anomalías en el funcionamiento de los equipos radiológicos, Radioprotección, 41 (11), 1-8.

Alcaraz, M. (2005). Evolución de la Protección Radiológica y Control de Calidad en la Radiología Dental española (1996-2001). Murcia: Servicio de Publicaciones de la Universidad de Murcia.

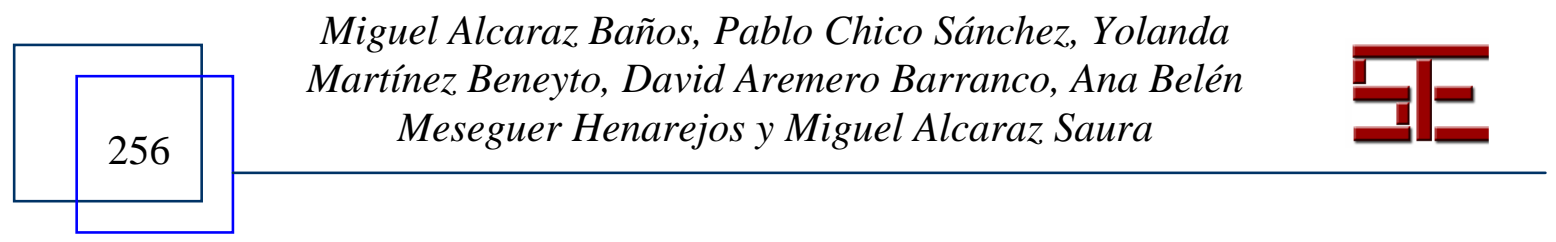




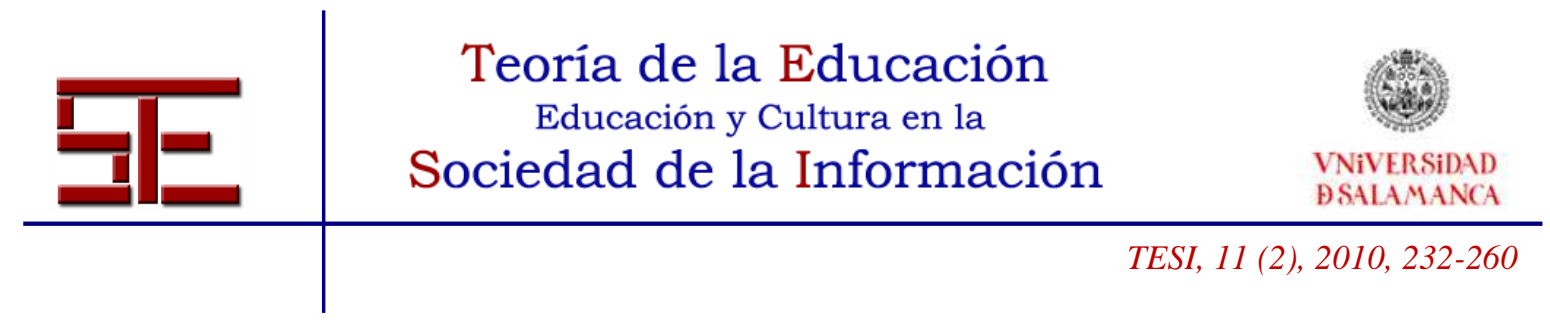

Alcaraz, M., Navarro, C.; Vicente, V. Y Canteras, M. (2006a). Dose reduction of intraoral dental radiography in Spain, Dentomaxillofacial Radiology, 35, 295-298.

Alcaraz, M., Chico, P., Armero, D., Saura Iniesta, A.M., Navarro, J. L. Y VICENTE, V. (2006b). Protección Radiológica y garantía de calidad en radiodiagnóstico (4a edición): Teleenseñanza a través de Internet en Ciencias de la Salud, Simposium Internacional sobre Protección del Paciente, Radioprotección, 49 (13), 212-214.

Alcaraz, M. (2008). La Radiología Dental en España. Murcia, Colección Editum, Murcia Servicio de Publicaciones de la UMU-Consejo de Seguridad Nuclear.

Alcaraz, M., García-Vera, M.C., Bravo, L.A., Martínez Beneyto, Y., Armero, D., Morent, J.J. Y Canteras, M. (2009a). Collimator with filtration compensator: clinical adaptation to meet European Union Recommendation 4F on radiological protection for dental radiography, Dentomaxillofac Radiol, 38 (6) ,413-20.

Alcaraz, M.; Parra, C.; Martínez, Y., Velaso, E. Y Canteras, M. (2009a). Is it true that the radiation dose to which patients are exposed has decreased with modern radiographic films? Dentomaxillofacial Radiol, 38, 92-97.

Alcaraz -Baños, M., Parra-Perez, C., Armero-Barranco, D., Velasco-Hidalgo, F. Y Velasco-Hidalgo, E. (2009a). Changes in radiological protection and quality control in Spanish dental installations: 1996-2003, Med Oral Patol Oral Cir Bucal, 14 (10), 499-505.

Alcaraz, M., Acevo, C., Castillo, J., Benavente-García, O., Armero, D., Vicente, V. Y Canteras, M. (2009d). Liposoluble antioxidants provide an effective radioprotective barrier. Br J Radiol, 82 (979),605-9.

Alcaraz, M., Velasco, E., Mertínez-Beneyto, Y., Velasco, F., Armero, D. Parra, C. Y Canteras, M. (2010). The status of Spain's dental practice following the European Union directive concerning radiological installations: eleven years on (1996-2007). Dentomaxillofacial Radiology (aceptado en prensa).

Añel, M.E. (2009): Revista de Medios y Comunicación Píxel Bit [online]. Cited 2009 Mayo1. Available from: http://www.sav.us.es/pixelbit

Castillo, J., Benavente-García, J., Del Baño, J.M., Lorente,J., Alcaraz, M. Y Dato, M.J. (2002). Radioprotective effects against to chromosomal damage induced in Human Lymphocytes by X-rays as a function of polymerisation grade of grape seed extracts, Journal of Medicinal Food, 4 (2), 117-123.

Del Baño, Mj., Castillo, J., Benavente-García, O., Lorente, J., Martín-Gil, R., Acevedo, C. Y Alcaraz ,M. (2006). Radioprotective Effects against to Chromosomal Damage Induced in Human Lymphocytes by $\gamma$-rays of Rosemary Phenolics: Carnosic Acid, Carnosol and Rosmarinic Acid, Journal of Agricultural and Food Chemistry, 54, 2064-2068.

EUROPEAN COMISSION (2002): Guidelines on Education and training in radiation protection for medical exposures. Radiation Protection $\mathrm{n}^{\circ}$ 116. European Commission. Available from: http://ec.europa.eu/energy/nuclear/radiation_protection/doc/publication/116.pdf,

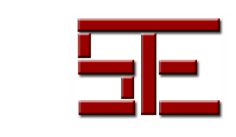

Miguel Alcaraz Baños, Pablo Chico Sánchez, Yolanda Martínez Beneyto, David Aremero Barranco, Ana Belén Meseguer Henarejos y Miguel Alcaraz Saura 


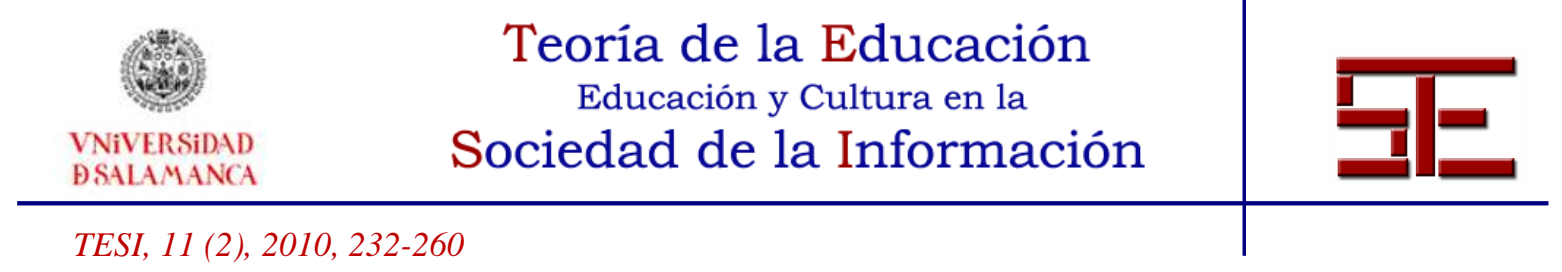

Fleetwood, J., Vaught, W., Feldman, D., Gracely, D., Kassutto, Z., Novack, D.A. (2002). Computed based learning program in Medical Ethics and Communication skills, Med Eth Ex Online, Teaching and Learning in Medicine, 12 (2), 96-104.

Friedman, C.P (1994). Coping with innovation frenzy, Acad Med, 69, 194-195.

Golsman, J., Blake, J. (1996). Multimedia courseware: transforming the classroom, Comput Nurs, 14 (5), 287-295.

Gunderman, R.B., Kaang, Y.P., Traley, R.E., Williamson, K.B. (2001). Instructional technology and radiology education, Radiology, 221 (1), 1-4.

Holt, N.F. (2001). Medical students need more radiology education, Acad Med, 76, 1.

Keane, D.R., Norman, G.R., Vickers, J. (1991). The inadequacy of recent research on computer assisted instruction, Acad Med, 66, 444-448.

Letterieg.S. (2003). Medical education as a science: the quality of evidence for computer-assisted instruction, Am J Obstet Gynecol, 188 (3), 847-853.

Lieberman, G., Abramson, R., Volkan, K., Mcardle, P.J. (2002). Tutor versus computer: a prospective comparison of interactive tutorial and computer-assisted instruction in radiology education, Acad Rad, 9 (1), 40-49.

Martínez-Beneyto, M., Alcaraz, M., Pérez-Lajarin, P., .Jodar, S., Chiva, F. Y Canteras, M. (2004). Dosis media de radiación ionizante por radiología dental intraoral en la práctica privada española, Archivos de OdontoEstomatología, 20 (2), 122-130.

Martínez-Beneyto, Y., Alcaraz-Baños, M., Pérez-Lajarin, L. Y Rushton, V.E. (2007). Justificación clínica para la utilización de radiología dental en un paciente adulto: una revisión de la literatura. Med Oral Pathol Oral Cir Bucal, 12 (3), 244-251.

Martínez-Beneyto, Y., Camacho-Alonso, F., Alcaraz-Baños, M., López-Jornet, P. Y Pérez-Lajarin, L. (2008). Spanish dental hygienist attitudes to oral radiology: assessment of a one-day pilot course. International Journal of Dental Hygiene, 6 (1), 13-18.

Navarro, J-L., Alcaraz-Baños, M., Gómez-Moraga, A., Vicente, V. Y Canteras, M. (2004). Ausencia de daño cromosómico y genotóxico inducido por la dosis de radiación administrada en las exploraciones gammagráficas, Revista Española de Medicina Nuclear, 23 (3), 174-82.

Pelayo-Alvarez. M., Albert-Ros, X., Gil-Latorre. F. Y Gutiérrez-Sigler, D. (2000). Feability analysis of a personalized training plan for learning research methodology, Medical Education, 34 (2), 139-145.

Rouse, D.P. (1999). Creating A Interactive Multimedia Computer Assisted Instruction, Program Comput Nurs, 17 (4), 171-176.

Sánchez-Campillo, M., Gabaldon, J.A., Castillo, J., Benavente-García, O., Del Baño, M.J., Alcaraz, M., Vicente, V., Alvarez, N. Y Lozano, J.A. (2009). Rosmarinic acid, aphoto protective agent against UV and other ionizing radiations, Food Chem Toxicol, 47 (2), 386-92.

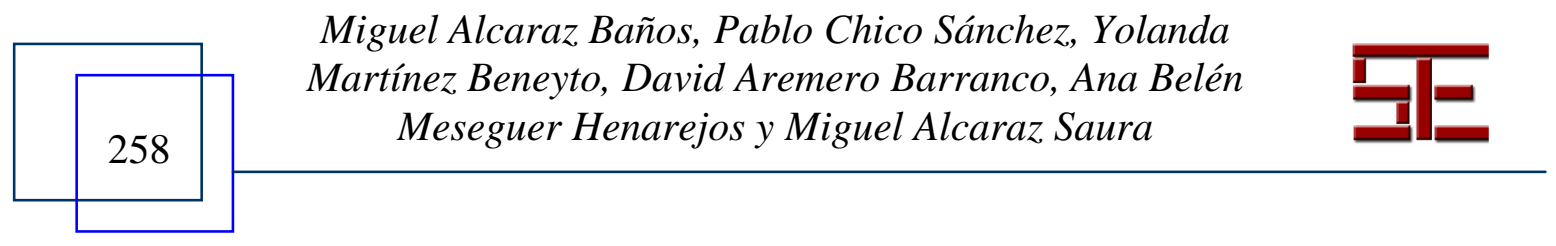




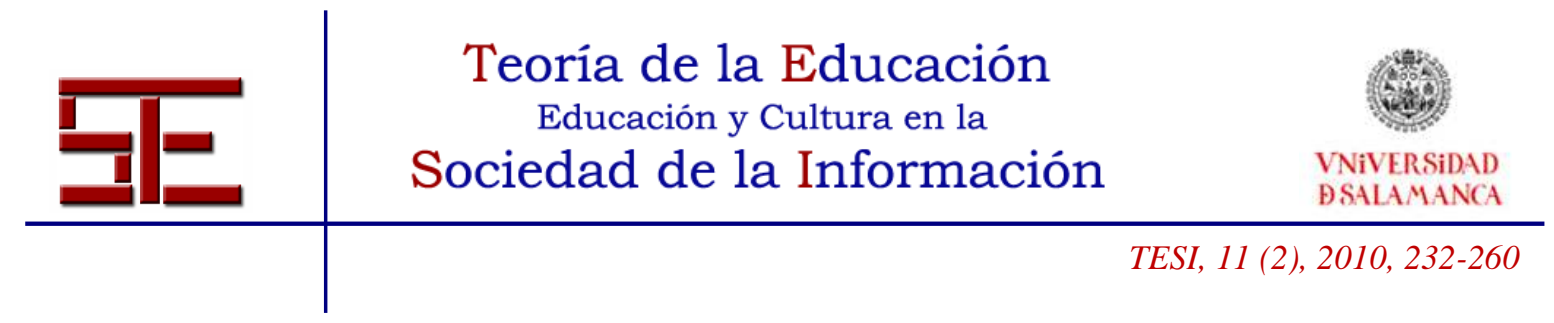

Sánchez-Llorente, J.M. (2009). Diseño, desarrollo, implementación y evaluación de una plataforma multicanal (L.M.S.) de apoyo al proceso de enseñanza aprendizaje "online" de la Física Médica. Tesis Doctoral, Universidad de Salamanca.

Squire, L.N., Becker, J.A. (1975). On the importance of teaching undergraduate radiology, Radiology, 117, 227-8.

Subramaniam, R.M., Gibson, R.N. (2007). Radiology teaching: essentials of a quality teaching programme, Australian Radiology, 51 (1), 42-45.

Torales Chaporro, O. E. (2008). Diseño y evaluación de una aplicación multimedia para la enseñanza de radiología a alumnos de medicina (AMERAM).Tesis Doctoral. Universidad de Málaga.

\section{Figuras.}

- Fig.1: Material de partida en la elaboración de los nuevos recursos y materiales (Alcaraz y Genovés, 1995).

- Fig.2: Materiales generados consecuencia de la subvención del Ministerio de Educación y Ciencia (Alcaraz, 2003).

- Fig.3: Libros editados mediante la subvención del Consejo de Seguridad Nuclear y que forman parte de los contenidos e iconografía desarrollada para el curso de teleenseñanza (Alcaraz, 2003, 2008).

- Fig.4.: Iconografía específica diseñada sobre test y ensayos de control de calidad y detectores de radiación ionizante elaborados específicamente para el desarrollo del curso de tele-enseñanza.

- Fig.5: Iconografía sobre errores y defectos habituales desarrolladas para los cursos de tele-enseñanza: elementos metálicos, joyas, exposiciones corporales totales ("niñografía") y entrada de luz en los chasis.

- Fig.6: Aspectos más significativos de los resultados de la autoevaluación de los alumnos, de la coevaluación alumnos-profesores y autoevaluación de la tarea realizada por el profesor.

- Fig.7: Página de entrada al curso con solicitud de claves de acceso al mismo.

- Fig.8: Página principal del curso en las primeras ediciones que te dirige a cada uno de los apartados del mismo y a los ejercicios de autoevaluación del alumno y evaluación del profesor.

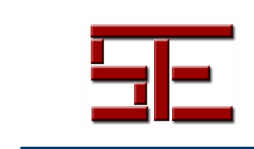

Miguel Alcaraz Baños, Pablo Chico Sánchez, Yolanda Martínez Beneyto, David Aremero Barranco, Ana Belén Meseguer Henarejos y Miguel Alcaraz Saura 


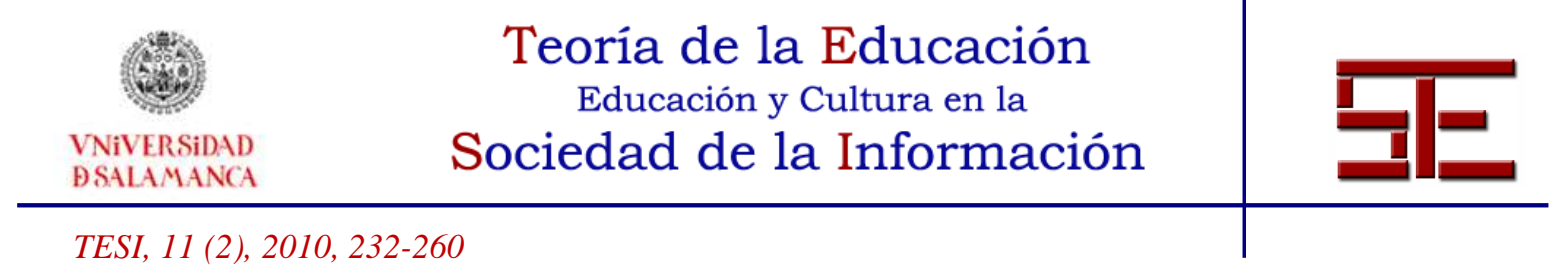

- Fig.9: Página de publicaciones y recursos complementarios a los contenidos evaluables durante en el formato utilizado durante las 5 primeras ediciones.

- Fig.10: Página actual de entrada al Curso de tele-enseñaza que se encuentra ofertado con acceso libre en la página http://webs.um.es/mab/miwiki/doku.php?id=docencia de la Universidad de Murcia. Sus materiales y la mayoría de sus recursos están disponibles libremente, aunque las ediciones oficiales sólo se realizan durante los períodos aprobados por la Universidad de Murcia.

- Fig.11: Contenidos evaluables del curso en correspondencia con las "Guidelines on Education and training in radiation protection for medical exposures. Radiation Protection $\mathrm{n}^{\mathrm{o}}$ 116" (European Commission 2000).

- Fig.12: Diploma oficial acreditativo de el aprovechamiento del curso de teleenseñanza.

Para citar el presente artículo puede utilizar la siguiente referencia:

Alcaraz Chico, M., Chico Sánchez, P., Martínez Beneyto, Y., Armero Barranco, D., Meseguer Henarejos, A.B. y Alcaraz Saura, M. (2010). Tele-enseñanza a través de Internet: la protección radiológica en Ciencias de la Salud, en Juanes Méndez, J.A. (Coord.) Avances tecnológicos digitales en metodologías de innovación docente en el campo de las Ciencias de la Salud en España. Revista Teoría de la Educación: Educación y Cultura en la Sociedad de la Información. Vol. 11, $\mathrm{n}^{\circ}$ 2. Universidad de Salamanca, pp. 232-260 [Fecha de consulta: dd/mm/aaaa]. http://campus.usal.es/ revistas_trabajo/index.php/revistatesi/article/view/7079/7112

Miguel Alcaraz Baños, Pablo Chico Sánchez, Yolanda Martínez Beneyto, David Aremero Barranco, Ana Belén Meseguer Henarejos y Miguel Alcaraz Saura 